\title{
ON THE ORIGIN OF MEMBRANE POTENTIAL IN MEMBRANES WITH POLARIZABLE NANOPORES
}

\author{
Ilya I. Ryzhkov ${ }^{a, b 1}$, Denis V. Lebedev ${ }^{a}$, Vera S. Solodovnichenko ${ }^{a}$, \\ Andrey V. Minakov ${ }^{b}$, and Mikhail M. Simunin ${ }^{a, b}$ \\ ${ }^{a}$ Institute of Computational Modelling SB RAS, Federal Research Center KSC SB RAS \\ Akademgorodok,660036 Krasnoyarsk, Russia,E-mail: rii@icm.krasn.ru \\ ${ }^{b}$ Siberian Federal University, Svobodny 79, 660041 Krasnoyarsk, Russia
}

\begin{abstract}
We report a new mechanism for the generation of membrane potential in polarizable nanoporous membranes separating electrolytes with different concentrations. The electric field generated by diffusion of ions with different mobilities induces a non-uniform surface charge, which results in charge separation inside the nanopore. The corresponding Donnan potentials appear at the pore entrance and exit leading to a dramatic enhancement of membrane potential in comparison with an uncharged non-polarizable membrane. At high concentration contrast, the interaction between electric field and uncompensated charge at a low concentration side results in the development of electrokinetic vortices. The theoretical predictions are based on the Space-Charge model, which is extended to nanopores with polarizable conductive surface for the first time. This model is validated against full Navier-Stokes, Nernst-Planck, and Poisson equations, which are solved in a high aspect ratio nanopore connecting two reservoirs. The experimental measurements of membrane potential of dielectric and conductive membranes in $\mathrm{KCl}$ and $\mathrm{NaCl}$ aqueous solutions confirm the theoretical results. The membranes are prepared from Nafen nanofibers with $10 \mathrm{~nm}$ in diameter and modified by depositing a conductive carbon layer. It is shown theoretically that the enhancement effect becomes greater with decreasing the electrolyte concentration and pore radius. A high sensitivity of membrane potential to the ratio of ion diffusion coefficients is demonstrated. The described phenomenon may find applications in precise determination of ion mobilities, electrochemical and bio-sensing, as well as design of nanofluidic and bioelectronic devices.
\end{abstract}

Keywords: membrane potential, diffusion potential, polarizable nanopore, induced charge, Space-Charge model

\section{Introduction}

The phenomenon of membrane potential at zero current refers to the appearance of potential difference between two electrolyte solutions with different concentrations separated by a membrane. In charged membranes, this difference arises due to Donnan equilibrium between diffusion and electric forces at membrane/solution interfaces (Donnan potentials), and electric field generated by diffusion of ions with different mobilities (diffusion potential) [1]. The measurement of membrane potential is used for characterizing the ionic permselectivity of ion exchange membranes and determining their charge density $[2,3]$. It also provides the basis for potentiometric ion sensing with the help of ion selective electrodes [4]. The membrane potential is a measure of maximum power that can be generated in reversed electrodialysis $[5,6]$. The resting potential difference across biological cell membranes regulates various cellular activities, such as proliferation and differentiation $[7,8]$.

The simplest and widely used theoretical approach for describing the membrane potential is the Teorell-Meyer-Sievers (TMS) model $[9,10]$. It assumes that the Debye screening length is larger than the pore size, so the potential and ion concentrations in the pore cross-section

\footnotetext{
${ }^{1}$ Corresponding author
} 
are uniform. In addition, the osmotic water transport is neglected. For densely charged membranes, the predictions of TMS model are accurate when the concentration difference across the membrane is small [11]. The TMS model was extended to include the finite ion size and dielectric exclusion effects in [12]. It was shown that they increase the membrane potential at a given concentration. The appearance of a maximum in the potential response of modified gold nanopores at high concentration contrast was successfully predicted by the two-dimensional model based on the Nernst-Planck and Poisson equations [13]. The Space-Charge (SC) model, which takes into account the radial variation of potential and ion concentrations as well as water transport in cylindrical pores, was first suggested in [14]. This model was revisited recently [15] by providing an essential simplification of working formulas. The experimental verification of SC model was performed by comparing the predicted streaming potential, pore conductivity, and membrane potential at zero current with the measured data [16]. The comparison between SC and TMS model showed that the latter overestimates the value of membrane potential for large surface charge and/or pore size exceeding the Debye length [17]. A similar conclusion was obtained for multivalent electrolytes [19]. The variation of membrane potential with $\zeta-$ potential was studied on the basis of SC model in [18]. The idea of obtaining information about the pore network morphology from membrane potential measurements was suggested in [20]. Using the SC model, a map relating the pore radius and volume charge density with the membrane potential was constructed. The effect of solution $\mathrm{pH}$ on membrane potential was investigated theoretically by the corresponding extension of SC model [21].

The measurement of membrane potential was used for determining the charge of commercial nanofiltration membranes [22] as well as laboratory made membranes [23] on the basis of TMS model. The permselectivity of commercial ion-exchange membranes in different electrolytes was studied by measuring their potential in [24]. It was shown that the binding affinity between counter-ions and fixed charge groups in the polymer can affect the permselectivity. The effect of unstirred layers adjacent to the membrane on the membrane potential was investigated in [25]. The thickness of the unstirred layer obtained from the limiting current density method was comparable with a value calculated as a parameter to reproduce the observed membrane potential.

In recent decades, a lot of research has been focused on the design of membranes with tunable ion transport. The latter can be realized by combining the pore design strategy (geometry and surface chemical modification) with external stimuli, such as transmembrane potential, solution pH, temperature, light, etc. [26-28]. A new class of membranes containing gold nanotubules that span a complete thickness of a porous polymeric support was suggested in [29]. Using membrane potential measurement at zero current, it was shown that their selectivity can be reversible switched from anion to cation by changing the potential applied to the conductive membrane surface. The membrane transport experiments revealed that the fluxes of anionic and cationic permeate species change as a function of applied potential [30,31]. The modulation of membrane potential by the external electric field in solid-state channels was also demonstrated in [32]. The theory of ion transport in electrochemically switchable conductive membranes based on a number of restrictive assumptions showed only satisfactory agreement with the experimental data [33]. It was shown in [34] that a qualitative similarity between predicted and measured membrane potential can be obtained by assuming that the applied voltage gives rise to a fixed charge proportional to it.

To correctly describe the ion transfer in nanopores with conductive surface, the fixed surface potential should be assumed [35]. Note that the electric field generated by moving ions near a conductive surface can induce polarization charges [36], which may in turn alter the pore transport characteristics. The induced-charge electrokinetic phenomena are actively investigated nowadays due to potential applications in microfluidic pumping and mixing [37], particle manipulation [38], and capacitive deionization [39]. The induction of surface charge by external electric field was employed for realizing nanopores with ion current rectification [40]. It was 
shown theoretically in [41] that the combination of nanochannel geometry and induced-charge effect results in cation (anion) permselectivity for forward (reversed) electric bias. Despite a growing number of studies in the area, a systematic investigation of membrane potential in membranes with conductive polarizable nanopores has not been performed neither theoretically nor experimentally. It is clear that the TMS and SC models with constant volume/surface charge cannot adequately describe this configuration.

In this paper, we report a new mechanism for the generation of membrane potential in polarizable conductive membranes via induced surface charge. The latter appears in electric field generated by diffusion of ions with different mobilities. It is shown theoretically and experimentally that this effect leads to a dramatic enhancement of diffusion potential in uncharged polarizable membranes in comparison with their non-polarizable counterparts. Theoretical predictions on the basis of Space-Charge model extended to polarizable nanopores are supported by the experimental measurements in dielectric and conductive membranes.

The paper is organized as follows. The mathematical models are presented in Section 2, while the membrane preparation and characterization as well as potential measurement are described in Section 3. Comparison between theory and experiment and discussion of the impact of various parameters on membrane potential is presented in Section 4. The main findings are summarized in Conclusion.

\section{Theoretical}

\subsection{The Teorell-Meyer-Sievers model for uncharged membrane}

Let us consider a porous membrane, which separates two reservoirs denoted by $L$ (left) and $R$ (right). The reservoirs contain aqueous solutions of the same monovalent and symmetric (1:1) electrolyte with concentrations $C_{L}$ and $C_{R}$, respectively $\left(C_{L}>C_{R}\right)$. In what follows, the ion concentrations will be used assuming that the activity coefficients are equal to unity.

We start with the case of uncharged membrane. The TMS theory for diffusion potential is based on the Nernst-Planck equation, the condition of electroneutrality, and equality of ion fluxes (i.e. zero current):

$$
\begin{aligned}
& J_{ \pm}=-D_{ \pm} \frac{d C_{ \pm}}{d Z} \mp \frac{D_{ \pm} F}{R_{g} T} C_{ \pm} \frac{d \Phi}{d Z}, \\
& C_{+}=C_{-}, \\
& J_{+}=J_{-}=\text {const. }
\end{aligned}
$$

Here $Z$ is the space coordinate along membrane thickness, $J_{ \pm}$are the ion fluxes, $C_{ \pm}$are the ion concentrations, $D_{ \pm}$are the ion diffusion coefficients, $\Phi$ is the electric potential, $R_{g}$ is the ideal gas constant, $T$ is the temperature, and $F$ is the Faraday constant. The boundary conditions imposed at the membrane entrance and exit are as follows

$$
Z=0: \quad C_{ \pm}=C_{L}, \quad \Phi=\Phi_{L} ; \quad Z=L_{p}: \quad C_{ \pm}=C_{R},
$$

where $L_{p}$ is the membrane thickness, and $\Phi_{L}$ is taken zero for simplicity.

Substituting the fluxes from (1) into (3) and taking into account (2), we can express the potential gradient $d \Phi / d Z$ from the resulting equation. Integration of this equation from the pore entrance $Z=0$ to the position $Z$ using boundary conditions (4) gives

$$
\Phi=\frac{R_{g} T}{F} \frac{D-1}{D+1} \ln \frac{C_{L}}{C_{ \pm}},
$$


where $D=D_{+} / D_{-}$is the ratio of ion diffusion coefficients. The substitution of (5) into (1) and integration of the resulting equations with boundary conditions (4) leads to

$$
C_{ \pm}=C_{L}+\frac{C_{R}-C_{L}}{L_{p}} Z, \quad J=2 J_{+}=2 J_{-}=4 \frac{C_{L}-C_{R}}{L_{p}}\left(\frac{1}{D_{+}}+\frac{1}{D_{-}}\right)^{-1} .
$$

Here $J=J_{+}+J_{-}$is the total ion flux.

The membrane potential is calculated as $\Delta \Phi=\Phi_{R}-\Phi_{L}=\Phi\left(L_{p}\right)$, so

$$
\Delta \Phi=\Delta \Phi_{D}=\frac{R_{g} T}{F} \frac{D-1}{D+1} \ln \frac{C_{L}}{C_{R}} .
$$

When $D_{+}<D_{-}$, the faster diffusing anion generates an electric field directed from reservoir with a higher concentration $(L)$ to that with a lower concentration $(R)$. It speeds up the cation and slows down the anion to make the ion fluxes equal. In this case, $\Delta \Phi_{D}<0$. When $D_{+}>D_{-}$, the direction of electric field is opposite, and one has $\Delta \Phi_{D}>0$. When $D \rightarrow 0$ or $D \rightarrow \infty$, the diffusion potential $\Delta \Phi_{D} \rightarrow \Phi_{I}^{+}$or $\Delta \Phi_{D} \rightarrow \Phi_{I}^{-}$, respectively. Here

$$
\Delta \Phi_{I}^{ \pm}= \pm \frac{R_{g} T}{F} \ln \frac{C_{L}}{C_{R}}
$$

is the Nernst potential of membrane, which transport only cations (plus sign) or anions (minus sign).

In what follows, we will also need the pore-averaged potential defined by

$$
\widetilde{\Phi}=\frac{1}{L_{p}} \int_{0}^{L_{p}} \Phi d Z=\frac{R_{g} T}{F} \frac{D-1}{D+1}\left(1+\frac{\ln \left(C_{L} / C_{R}\right)}{1-C_{L} / C_{R}}\right) .
$$

It should be noted that the accurate treatment of diffusion potential generation requires consideration of time-dependent problem [42]. The solution presented here is an approximation, which is valid at times much larger than the time of diffusion over the Debye length (typically $\left.10^{-9} \mathrm{~s}\right)$.

\subsection{The Teorell-Meyer-Sievers model for charged membrane}

When a membrane is uniformly charged, the electroneutrality condition (2) is replaced by $C_{+}-C_{-}+X=0$, where $X$ is the volume charge density. For a cylindrical pore of radius $R_{p}, X$ can be related to the surface charge density $\sigma$ by $X=2 \sigma / F R_{p}$. The presence of charge results in the appearance of Donnan potentials at the pore entrance and exit [11]:

$$
\begin{gathered}
\Delta \Phi_{L}=\Phi(0)-\Phi_{L}=\frac{R_{g} T}{F} \ln \left(\frac{X+\sqrt{X^{2}+4 C_{L}^{2}}}{2 C_{L}}\right), \\
\Delta \Phi_{R}=\Phi_{R}-\Phi\left(L_{p}\right)=-\frac{R_{g} T}{F} \ln \left(\frac{X+\sqrt{X^{2}+4 C_{R}^{2}}}{2 C_{R}}\right) .
\end{gathered}
$$

The derivation of diffusion potential for a charged membrane is performed similarly to that described in Section 2.1, while the details can be found in [11]. In the TMS theory, the total membrane potential is the sum of Donnan potentials and diffusion potential: $\Delta \Phi=$ $\Delta \Phi_{L}+\Delta \Phi_{R}+\Delta \Phi_{D}$. Thus, we arrive to the expression

$$
\Delta \Phi=\Phi_{R}-\Phi_{L}=\frac{R_{g} T}{F}\left[\ln \left(\frac{C_{R}}{C_{L}} \frac{X+\sqrt{X^{2}+4 C_{L}^{2}}}{X+\sqrt{X^{2}+4 C_{R}^{2}}}\right)+\mathcal{D} \ln \left(\frac{-\mathcal{D} X+\sqrt{X^{2}+4 C_{L}^{2}}}{-\mathcal{D} X+\sqrt{X^{2}+4 C_{R}^{2}}}\right)\right]
$$

where $\mathcal{D}=(D-1) /(D+1)$.

For a highly charged membrane (formally when $X \rightarrow \pm \infty$ ), the membrane potential tends to the Nernst potential (8), where the plus (minus) sign corresponds to the case of ideal cationselective (anion-selective) membrane. For an uncharged membrane $(X=0)$, the membrane potential (10) reduces to the diffusion potential (7). 


\subsection{The Navier-Stokes / Nernst-Planck / Poisson model}

When the Debye screening length is less or comparable with the pore radius, the electric double layers do not overlap. In this case, the TMS model fails to predict the membrane potential accurately. This model cannot also describe a membrane with conductive surface maintained at fixed potential, or ideally polarizable membrane floating in electric field. Such situations can be handled by considering the full Navier-Stokes, Nernst-Planck, and Poisson equations together with a pore model representing membrane structure.

The membrane is usually modelled as an array of cylindrical pores of length $L_{p}$ and radius $R_{p}$. A single pore connects two reservoirs of length $L_{r}$ and radius $R_{r}$ with electrolyte concentrations $C_{L}$ and $C_{R}$ (Fig. 1). The reservoirs are maintained at equal pressures. For a 1:1 electrolyte, the molar fluxes of ions are given by the extended Nernst-Planck equation:

$$
\boldsymbol{J}_{ \pm}=C_{ \pm} \boldsymbol{U}-D_{ \pm} \nabla C_{ \pm} \mp \frac{D_{ \pm} F}{R_{g} T} C_{ \pm} \nabla \Phi
$$

where $\boldsymbol{U}$ is the fluid velocity. For a two-dimensional axisymmetric case, $\boldsymbol{U}=(U, V)$, where $U$ is the radial velocity, and $V$ is the axial velocity. The components of ion fluxes in $Z$ direction are denoted by $J_{ \pm}$. Then $J=J_{+}+J_{-}$is the total ion flux, and $I=J_{+}-J_{-}$is the ion current in axial direction.

Let us introduce dimensionless radial and axial coordinates, velocity, pressure, ion concentrations, electric potential, and ion fluxes as follows:

$$
\begin{gathered}
R=R_{p} r, \quad Z=R_{p} z, \quad \boldsymbol{U}=\frac{D_{-}}{R_{p}} \boldsymbol{u}, \quad P=C_{0} R_{g} T p, \\
C_{ \pm}=C_{0} c_{ \pm}, \quad \Phi=\frac{R_{g} T}{F} \varphi, \quad \boldsymbol{J}_{ \pm}=\frac{D_{-} C_{0}}{R_{p}} \boldsymbol{j}_{ \pm} .
\end{gathered}
$$

Here $C_{0}$ is the reference concentration taken in this work as $C_{0}=1 \mathrm{mM}\left(1 \mathrm{~mol} / \mathrm{m}^{3}\right)$. The ion fluxes (11) are written in dimensionless form as follows:

$$
\boldsymbol{j}_{+}=c_{+} \boldsymbol{u}-D \nabla c_{+}-D c_{+} \nabla \varphi, \quad \boldsymbol{j}_{-}=c_{-} \boldsymbol{u}-\nabla c_{-}+c_{-} \nabla \varphi .
$$

The $z$ components of these fluxes are denoted by $j_{ \pm}$, so $j=j_{+}+j_{-}$and $i=j_{+}-j_{-}$are dimensionless total ion flux and ion current in axial direction, respectively. The dimensionless stationary Navier-Stokes, Nernst-Planck, and Poisson equations have the form

$$
\begin{aligned}
& \alpha \mathcal{R}(\boldsymbol{u} \cdot \nabla) \boldsymbol{u}=-\nabla p+\alpha \nabla^{2} \boldsymbol{u}-\left(c_{+}-c_{-}\right) \nabla \varphi \\
& \nabla \cdot \boldsymbol{u}=0 \\
& \boldsymbol{u} \cdot \nabla c_{+}=-\nabla \cdot D\left(-\nabla c_{+}-c_{+} \nabla \varphi\right), \\
& \boldsymbol{u} \cdot \nabla c_{-}=-\nabla \cdot\left(-\nabla c_{-}+c_{-} \nabla \varphi\right), \\
& \nabla^{2} \varphi=-\frac{1}{2 \lambda^{2}}\left(c_{+}-c_{-}\right) .
\end{aligned}
$$

The system includes the following dimensionless parameters:

$$
\mathcal{R}=\frac{\rho D_{-}}{\mu}, \quad \alpha=\frac{\mu D_{-}}{C_{0} R_{g} T R_{p}^{2}}, \quad D=\frac{D_{+}}{D_{-}}, \quad \lambda=\frac{1}{R_{p}} \sqrt{\frac{\varepsilon \varepsilon_{0} R_{g} T}{2 C_{0} F^{2}}},
$$

where $\mathcal{R}$ is the Reynolds number based on the velocity scale defined in (12), $\alpha$ is the dimensionless viscosity parameter, $D$ is the ratio of ion diffusion coefficients, and $\lambda$ is the dimensionless Debye length. In formulas (18), $\rho$ is the density of ionic solution, $\varepsilon$ is its relative permittivity, 


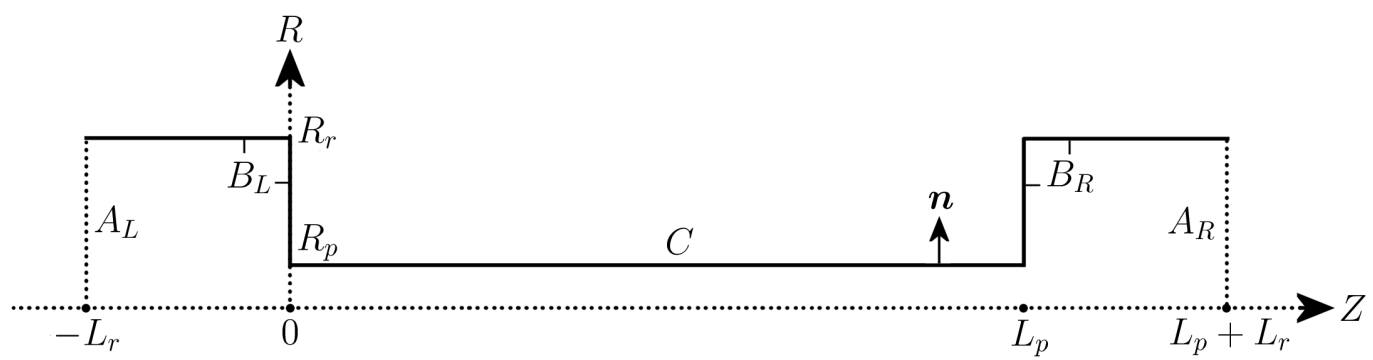

Figure 1: The geometry of the pore and reservoirs.

Table 1: The boundary conditions.

\begin{tabular}{lllll}
\hline \hline $\begin{array}{l}\text { Boundary } \\
\text { segment }\end{array}$ & $\begin{array}{l}\text { Navier-Stokes } \\
\text { equations }\end{array}$ & $\begin{array}{l}\text { Poisson } \\
\text { equation }\end{array}$ & $\begin{array}{l}\text { Nernst-Planck } \\
\text { equations }\end{array}$ & $\begin{array}{l}\text { Nernst-Planck eqns. } \\
\text { (Slotboom variables) }\end{array}$ \\
\hline$A_{L}$ & $p=0$ & $\varphi=0$ & $c_{ \pm}=c_{L}$ & $\widetilde{c}_{ \pm}=c_{L}$ \\
$A_{R}$ & $p=0$ & $\nabla \varphi \cdot \boldsymbol{n}=0$ & $c_{+}=c_{R}$ & $\widetilde{c}_{+}=c_{R} e^{\varphi_{R}}$ \\
& & $\boldsymbol{j}_{-} \cdot \boldsymbol{n}=\boldsymbol{j}_{+} \cdot \boldsymbol{n}$ & $\nabla \widetilde{c}_{-} \cdot \boldsymbol{n}=e^{-2 \varphi_{R}} D \nabla \widetilde{c}_{+} \cdot \boldsymbol{n}$ \\
$B_{L}, B_{R}$ & $\boldsymbol{u}=0$ & $\nabla \varphi \cdot \boldsymbol{n}=0$ & $\boldsymbol{j}_{ \pm} \cdot \boldsymbol{n}=0$ & $\nabla \widetilde{c}_{ \pm} \cdot \boldsymbol{n}=0$ \\
$C$ & $\boldsymbol{u}=0$ & $\mathrm{C} 1: \nabla \varphi \cdot \boldsymbol{n}=\sigma_{s}$ & $\boldsymbol{j}_{ \pm} \cdot \boldsymbol{n}=0$ & $\nabla \widetilde{c}_{ \pm} \cdot \boldsymbol{n}=0$ \\
& $\mathrm{C} 2: \varphi=\varphi s$ & \\
& $\mathrm{C} 3: \int_{s} \nabla \varphi \cdot \boldsymbol{n} d s=q_{s}$ \\
\hline \hline
\end{tabular}

and $\varepsilon_{0}$ is the vacuum permittivity. It should be noted that the flow in nanopores typically corresponds to low Reynolds numbers, so the convective term in (13) can be neglected. However, we have retained it for completeness.

The boundary conditions are summarized in Table 1 (see also Fig. 1). An outward unit normal to the boundary is denoted by $\boldsymbol{n}$. At the entrance to the left reservoir $A_{L}$, we impose zero pressure and potential, and specify the ion concentration $c_{L}=C_{L} / C_{0}$. At the exit from the right reservoir $A_{R}$, zero pressure and potential gradient as well as the concentration of cations $c_{R}=C_{R} / C_{0}$ are set. To ensure that the ion current is zero, the equality of anion and cation fluxes is imposed at the boundary $A_{R}$. The calculations show that the boundary condition for anion concentration $c_{-}=c_{R}$ at $A_{R}$ is automatically satisfied in this case. However, when both concentrations $c_{ \pm}=c_{R}$ are specified at $A_{R}$, the resulting ion current becomes non-zero [43]. The no-slip and impermeability conditions are imposed at the walls $B_{L}, B_{R}$, and $C$. In addition, the reservoir walls $B_{L}$ and $B_{R}$ are not charged.

In this work, we consider three different boundary conditions at the pore wall: (C1) constant surface charge density $\sigma ;(\mathrm{C} 2)$ constant surface potential $\Phi_{s}$; (C3) constant total surface charge $Q_{s}$. The corresponding dimensionless quantities are introduced by

$$
\sigma_{s}=\frac{\sigma}{\varepsilon \varepsilon_{0} R_{g} T / F R_{p}}, \quad \varphi_{s}=\frac{\Phi_{s}}{R_{g} T / F}, \quad q_{s}=\frac{Q_{s}}{\varepsilon \varepsilon_{0} R_{g} T R_{p} / F} .
$$

In case $\mathrm{C} 3, Q_{s}$ characterizes the total charge acquired by the conducting polarizable surface due to physicochemical mechanism. The electric field applied externally or formed inside the pore due to movement of ions causes the redistribution of surface charge. It is convenient to 
introduce the dimensional $\bar{\sigma}$ and dimensionless $\bar{\sigma}_{s}$ total surface charge densities according to

$$
\bar{\sigma}=\frac{Q_{s}}{2 \pi R_{p} L_{p}}, \quad \bar{\sigma}_{s}=\frac{q_{s}}{2 \pi L_{p} / R_{p}} .
$$

The floating boundary condition C3 is realized by solving the problem with constant potential at the pore wall. The wall potential is found in order to satisfy the condition of constant total surface charge.

In what follows, we will need the quantities averaged over the pore cross-section. In particular, the dimensional average axial velocity is defined by

$$
\bar{V}=\frac{2}{R_{p}^{2}} \int_{0}^{R_{p}} V R d R .
$$

The average pressure $\bar{P}$, ion concentrations $\bar{C}_{ \pm}$, potential $\bar{\Phi}$, axial ion fluxes $\bar{J}_{ \pm}$, total axial ion flux $\bar{J}=\bar{J}_{+}+\bar{J}_{-}$, and axial ion current $\bar{I}=\bar{J}_{+}-\bar{J}_{-}$are introduced in the same way. The corresponding dimensionless quantities are $\bar{v}, \bar{p}, \bar{c}_{ \pm}, \bar{\varphi}, \bar{j}_{ \pm}, \bar{j}=\bar{j}_{+}+\bar{j}_{-}$, and $\bar{i}=\bar{j}_{+}-\bar{j}_{-}$.

The membrane potential is calculated as a difference between the average potential values at the reservoir boundaries $A_{R}$ and $A_{L}$ (see Fig. 1):

$$
\Delta \Phi=\bar{\Phi}_{R}-\bar{\Phi}_{L}=\bar{\Phi}_{R}
$$

An alternative formulation of governing equations can be derived by introducing the Slotboom variables according to [44-46]

$$
c_{ \pm}=e^{\mp \varphi} \widetilde{c}_{ \pm}
$$

Using these variables, equations (13)-(17) are rewritten as

$$
\begin{aligned}
& \alpha \mathcal{R}(\boldsymbol{u} \cdot \nabla) \boldsymbol{u}=-\nabla p+\alpha \nabla^{2} \boldsymbol{u}-\left(e^{-\varphi} \widetilde{c}_{+}-e^{\varphi} \widetilde{c}_{-}\right) \nabla \varphi \\
& \nabla \cdot \boldsymbol{u}=0 \\
& \nabla \cdot\left(e^{-\varphi}\left(\boldsymbol{u} \widetilde{c}_{+}-D \nabla \widetilde{c}_{+}\right)\right)=0, \\
& \nabla \cdot\left(e^{\varphi}\left(\boldsymbol{u} \widetilde{c}_{-}-\nabla \widetilde{c}_{-}\right)\right)=0, \\
& \nabla^{2} \varphi=-\frac{1}{2 \lambda^{2}}\left(e^{-\varphi} \widetilde{c}_{+}-e^{\varphi} \widetilde{c}_{-}\right) .
\end{aligned}
$$

After the transformation, the boundary conditions for Navier-Stokes and Poisson equations remain the same, while the conditions for the Nernst-Planck equations are transformed into a simpler form, which is given in the last column of Table 1 . The quantity $\varphi_{R}$ is the value of dimensionless potential at the boundary $A_{R}$. The use of Slotboom variables has a number of advantages in comparison with the use of primitive variables [46]. First, the equations for ion concentrations and boundary conditions involving ion fluxes are simplified. Second, the Slotboom formulation provides more robust convergence and a significant reduction of computational time (up to 10 times).

Numerical solution of two-dimensional governing equations is obtained with the help of ANSYS Fluent 14.5 (Academic version). The stationary problem is solved iteratively by the Coupled method in finite volume formulation. For floating boundary condition, an initial guess for the surface potential is set and adjusted at each iteration step. The characteristics of computational mesh are chosen in such a way as to ensure that further refinement does not influence the solution quality. The following dimensions of pore and reservoirs are used: $R_{p}=8$ $\mathrm{nm}, L_{p}=4000 \mathrm{~nm}, R_{r}=100 \mathrm{~nm}, L_{r}=400 \mathrm{~nm}$ (their choice is discussed further in Section 4.1). The number of nodes in radial and axial directions is 50 and 3000 for the nanopore, and 
130 and 150 for each reservoir, respectively. The mesh has clustering in the axial direction near the pore entrance and exit as well as in the reversed radial direction in the reservoirs. In the pore, the mesh is radially uniform. More details on the numerical implementation can be found in $[46]$.

The advantage of Navier-Stokes / Nernst-Planck-Poisson (NS-NPP) model lies in the fact that it can accurately describe the structure of electric double layer in the pore as well as the influence of osmotic flow on ion transport for a variety of boundary conditions (fixed surface charge / fixed surface potential / floating boundary). When the model equations are solved in the pore and reservoirs, the change of potential, ion concentrations, and pressure at the membrane/solution interface can be modelled in details. The results can be compared with those obtained from other models (TMS, SC, or their extensions) and validate them in a given range of parameters. The disadvantage of the described model is a large computational time (a few hours for a single case) and numerical instability for large aspect ratio pores (length / radius $>10^{3}$ ). Thus, this model cannot be used for determination of membrane characteristics by fitting the experimental data to theoretical predictions (e.g. membrane potential dependence the ratio of concentrations $C_{L} / C_{R}$ ).

\subsection{The Space-Charge model}

The Space-Charge (SC) model can be considered as a model with the best relation between accuracy and computational efficiency. It is derived from Navier-Stokes, Nernst-Planck, and Poisson equations by introducing several assumptions appropriate for large aspect ratio pores and using jump boundary conditions at the membrane/solution interface. Until now, this model has been used for describing pores with fixed surface charge only [14-20]. In this work, the SC model is extended to pores with fixed potential as well as floating boundaries for the first time. We do not repeat the model derivation, which was described earlier in details [15], but introduce proper modifications for new types of boundary conditions and propose the computational procedure.

The dimensionless variables for the SC model are introduced according to (12) with exceptions for axial coordinate, velocity, and ion fluxes, which are given by

$$
Z=L_{p} z, \quad \boldsymbol{U}=\frac{D_{-}}{L_{p}} \boldsymbol{u}, \quad \boldsymbol{J}_{ \pm}=\frac{D_{-} C_{0}}{L_{p}} \boldsymbol{j}_{ \pm} .
$$

The dimensionless potential $\varphi$, ion concentrations $c_{ \pm}$, and pressure $p$ are represented as

$$
\begin{gathered}
\varphi(r, z)=\phi_{v}(z)+\psi(r, z), \quad c_{ \pm}(r, z)=c_{v}(z) \exp (\mp \psi(r, z)), \\
p(r, z)=p_{v}(z)+2 c_{v}(z) \cosh (\psi(r, z)),
\end{gathered}
$$

Here the ion concentrations satisfy the Boltzmann distribution. The function $\psi$ satisfies the Poisson equation with boundary condition of axial symmetry

$$
\begin{gathered}
\frac{1}{r} \frac{\partial}{\partial r}\left(r \frac{\partial \psi(r, z)}{\partial r}\right)=\frac{c_{v}(z)}{\lambda^{2}} \sinh \psi(r, z), \\
\frac{\partial \psi}{\partial r}(0, z)=0 .
\end{gathered}
$$

The constant surface charge density is imposed by

$$
\frac{\partial \psi}{\partial r}(1, z)=\sigma_{s}
$$

while for the constant surface potential one should write

$$
\psi(1, z)=\varphi_{s}-\phi_{v}(z) .
$$


For a polarizable conductive pore wall, the surface potential $\varphi_{s}$ should be determined in order to satisfy the floating boundary condition

$$
\int_{0}^{1} \frac{\partial \psi}{\partial r}(1, z) d z=\bar{\sigma}_{s}
$$

It should be noted that when modelling the membrane potential in pores with constant $\zeta$-potential in [18], the boundary condition $\psi(1, z)=\zeta$ was used instead of (24). In this case, the total potential $\varphi$ at the pore wall was in fact non-constant since $\phi_{v}$ is zero at the pore entrance and equals to membrane potential at the pore exit (see conditions (30) below).

The phenomenological flux-force formalism is used to relate the average volume flux $\bar{v}$ (or average axial velocity), average ion flux $\bar{j}$, and average ion current $\bar{i}$ to the gradients of virtual pressure $p_{v}$, virtual chemical potential $\mu_{v}=\ln c_{v}$, and virtual electric potential $\phi_{v}$ :

$$
\left(\frac{d p_{v}}{d z}, \frac{d \mu_{v}}{d z}, \frac{d \phi_{v}}{d z}\right)^{T}=L(\bar{v}, \bar{j}, \bar{i})^{T}
$$

Here $L=-\mathcal{L}^{-1}$ is the symmetric $3 \times 3$ matrix. The coefficients of matrix $\mathcal{L}=\left\{\mathcal{L}_{i j}(z)\right\}$ depend on the function $\psi(r, z)$ and virtual concentration $c_{v}(z)$. They are given in Appendix.

The membrane potential is measured at zero current $(\bar{i}=0)$. In this case, system $(26)$ becomes

$$
\begin{aligned}
\frac{d p_{v}}{d z} & =L_{11} \bar{v}+L_{12} \bar{j}, \\
\frac{1}{c_{v}} \frac{d c_{v}}{d z} & =L_{12} \bar{v}+L_{22} \bar{j}, \\
\frac{d \phi_{v}}{d z} & =L_{13} \bar{v}+L_{23} \bar{j} .
\end{aligned}
$$

The corresponding boundary conditions are derived by assuming equal (zero) pressures and constant concentrations in the reservoirs, and setting the potential at the left reservoir to zero. By putting $\psi(r, z)=0$ in $(20)$, one arrives at

$$
\begin{aligned}
& z=0: \quad p_{v}=-2 c_{L}, \quad c_{v}=c_{L}, \quad \phi_{v}=0, \\
& z=1: \quad p_{v}=-2 c_{R}, \quad c_{v}=c_{R}, \quad \phi_{v}=\Delta \varphi .
\end{aligned}
$$

Here $\Delta \varphi$ is the dimensionless potential difference between the reservoirs (membrane potential).

Note that the use of boundary condition (24) and integral condition (25) introduces some difficulties. The surface potential $\varphi_{s}$ is not known in advance. The virtual potential $\phi_{v}(x)$ is found by integration of system (27) - (29) with coefficients $L_{i j}(z)$, which in turn depend on the solution $\psi(r, z)$ of problem (21), (22), (24), (25). It makes the system strongly coupled and non-linear.

Let us express

$$
d z=\frac{d c_{v}}{c_{v}\left(L_{12} \bar{v}+L_{22} \bar{j}\right)}
$$

from Eq. (28) and substitute it into Eq. (27) and (29). Integration of resulting equations over the pore length with the help of boundary conditions (30) gives

$$
\begin{gathered}
\int_{c_{L}}^{c_{R}} \frac{L_{11} \bar{r}+L_{12}}{c_{v}\left(L_{12} \bar{r}+L_{22}\right)} d c_{v}+2\left(c_{R}-c_{L}\right)=0, \\
\bar{j}=\int_{c_{L}}^{c_{R}} \frac{d c_{v}}{c_{v}\left(L_{12} \bar{r}+L_{22}\right)}, \\
\phi_{v}\left(c_{v}\right)=\int_{c_{L}}^{c_{v}} \frac{L_{13} \bar{r}+L_{23}}{c_{v}\left(L_{12} \bar{r}+L_{22}\right)} d c_{v}
\end{gathered}
$$


where $\bar{r}=\bar{v} / \bar{j}$ is the fluxes ratio. It follows from (21), (24), and (34) that one can write $\psi=\psi\left(r, c_{v}\right), \phi_{v}=\phi\left(c_{v}\right), L_{i j}=L_{i j}\left(c_{v}\right)$, see also Appendix.

The dimensionless axial velocity is determined by the formula

$$
\begin{gathered}
v(r, z)=\frac{r^{2}-1}{4 \alpha} \frac{d p}{d z}+\frac{2}{\alpha}\left(\ln r \int_{0}^{r} r^{\prime} \cosh \left(\psi\left(r^{\prime}, z\right)\right) d r^{\prime}+\int_{r}^{1} r^{\prime} \ln r^{\prime} \cosh \left(\psi\left(r^{\prime}, z\right)\right) d r^{\prime}\right) \frac{d c_{v}}{d z}+ \\
+\frac{2 \lambda^{2}}{\alpha}\left(\psi_{s}-\psi(r, z)\right) \frac{d \phi_{v}}{d z}
\end{gathered}
$$

where $\psi_{s}=\psi(1, z)$.

The calculation is performed as follows. For a non-polarizable dielectric pore with constant surface charge density $\sigma_{s}$, problem (21)-(23) is solved numerically for a set of successive values $c_{v}=c_{v k}, k=0, \ldots, n$, where $c_{v 0}=c_{L}, c_{v n}=c_{R}$. Then the ratio of fluxes $\bar{r}=\bar{v} / \bar{j}$ is found numerically from (32), and the ion flux $\bar{j}$ is obtained from (33). The potential difference between reservoirs $\Delta \varphi=\phi_{v}\left(c_{R}\right)$ is determined from (34), while the virtual variables are found by integration of (27)-(30).

For a polarizable conductive pore with constant total surface charge density $\bar{\sigma}_{s}$, an initial guess for the surface potential $\varphi_{s}$ is set. Here problem (21), (22), (24) is solved for each $c_{v k}$ at fixed $\varphi_{s}$ and $\bar{j}$. Note that $\phi_{v}\left(c_{v 0}\right)=\phi_{v}\left(c_{L}\right)=0$. The value $\phi_{v}\left(c_{v k}\right)$ is found iteratively starting from $\phi_{v}\left(c_{v, k-1}\right)$ and repeating the solution of $(21),(22),(24)$ followed by application of (34). The calculation is performed iteratively to find the fluxes ratio $\bar{r}$ from (32). Then $\bar{j}$ is calculated from (33) and virtual variables are obtained by integration of (27)-(30). It allows to find $\psi(r, z)=\psi\left(r, c_{v}(z)\right)$ and finally calculate the distribution of surface charge $\sigma(z)=\partial \psi / \partial r(1, z)$ and the integral in the left-hand side of (25). The whole procedure is iterated to find the surface potential $\varphi_{s}$, with which Eq. (25) is satisfied. This iteration is not required when the surface potential is fixed externally.

The integration of Poisson equation (21) is performed by reducing it to two first-order ODE and applying the Runge-Kutta-Merson method of 5th order starting from $r=\epsilon$ to $r=1$, where $\epsilon$ is close to zero. Here an additional boundary condition $\psi(\epsilon, x)=\psi_{0}$ is required. The value $\psi_{0}$ is determined by the shooting method in order to satisfy boundary condition (24) at fixed $z$. The initial approximation for it can be found from analytical solution derived in [47].

\section{Experimental}

\subsection{Membrane preparation}

To validate the predictions of theoretical model experimentally, we have synthesized membranes with dielectric as well as conductive nanoporous structure. In this paper, we briefly describe their preparation and characterization. More detailed information can be found in [48].

The membrane preparation technique is schematically shown in Fig. 2. The membranes are produced from Nafen ${ }^{\mathrm{TM}}$, which is supplied by ANF Technology in the form of blocks composed of highly aligned $\gamma$-phase alumina nanofibers with the diameter of 10-15 nm and length more than $100 \mathrm{~mm}$ [49]. Nafen nanofibers are dispersed in deionized water (the weight ratio of Nafen:water is 1:200). The suspension is agitated with a magnetic stirrer for 30 minutes followed by 15 minutes of ultrasonic treatment (Sonics \& Materials VC-505, USA). The alignment of Nafen fibers facilitates their separation from each other and allowes preserving relatively high aspect ratios $(\sim 100)$ after treatment. The obtained suspension is filtered through the rough Teflon filter (average pore size of $0.6 \mu \mathrm{m}$ ) and dried in air. The resulting membrane is sintered at $800{ }^{\circ} \mathrm{C}$ during 4 hours, which ensures its structural stability in aqueous solutions. Membranes are prepared in the form of circular discs with the diameter of around $40 \mathrm{~mm}$ and thickness of about $400 \mu \mathrm{m}$, Fig. 2 (b). 
$\mathrm{a}$
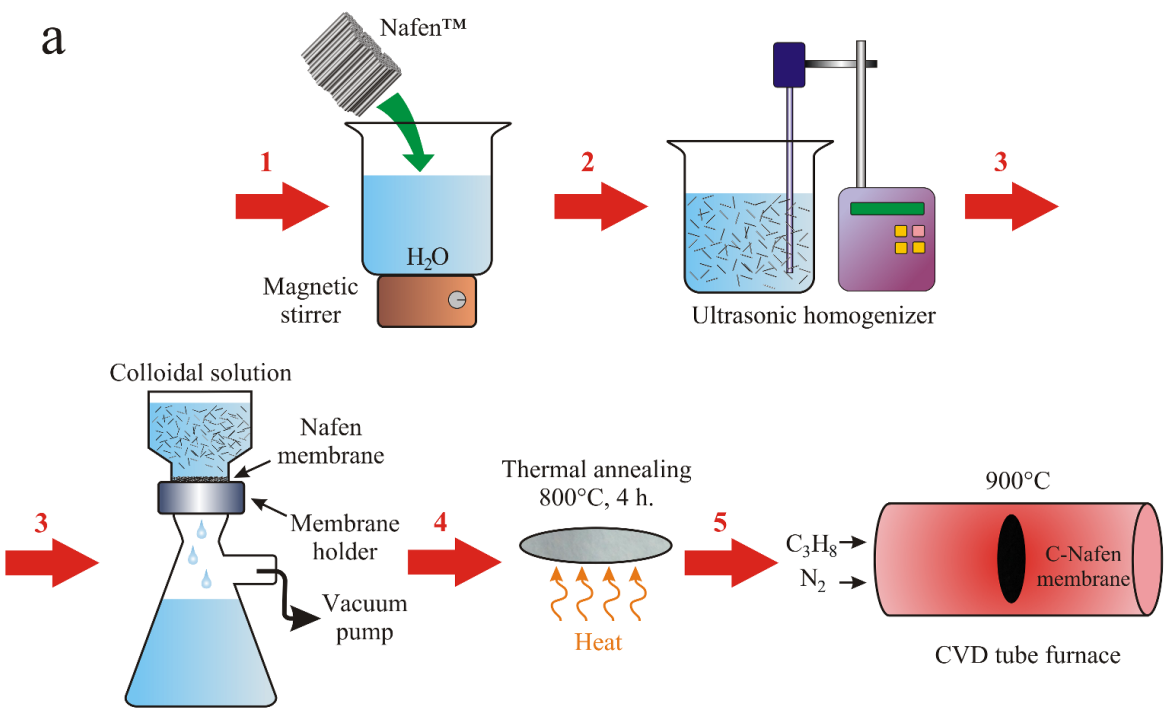
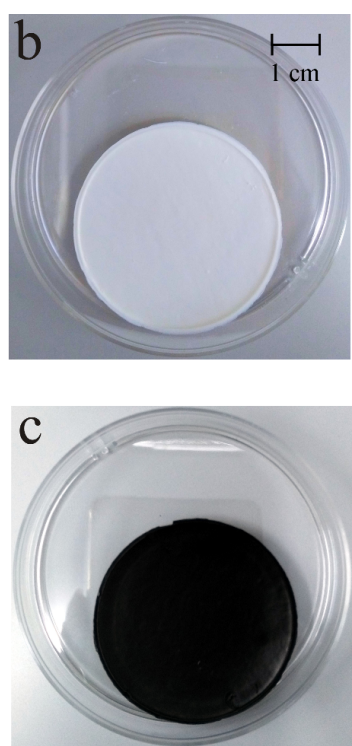

Figure 2: Membrane preparation technique (a), top view of Nafen membrane before (b) and after (c) deposition of carbon by CVD method.


Figure 3: SEM images of the membrane surface before (a) and after (b) deposition of carbon. The corresponding TEM images of nanofibers are shown in the inserts.

To form carbon layers on the membrane surface, chemical vapor deposition (CVD) is used. The synthesis of carbon layers is conducted in the homemade CVD reactor at $900{ }^{\circ} \mathrm{C}$ (heating rate of 20-30/min) in propane/nitrogen mixture (1/15) with the total flow rate of $4000 \mathrm{ml} / \mathrm{min}$ during 60 seconds. The mass gain after CVD is around $15 \%$. Then the sample is slowly cooled to $150{ }^{\circ} \mathrm{C}$ in the atmosphere of nitrogen. A typical view of the obtained membrane is shown in Fig. 2 (c). In what follows, the samples with and without deposited carbon layer will be referred to as $\mathrm{C}-\mathrm{Nafen}$ membrane and Nafen membrane, respectively.

It should be noted that the Nafen nanofibers were used for the preparation of ultrafiltration membranes with asymmetric structure in [50]. The fabrication of hybrid membranes by incorporating silica into alumina nanofiber scaffold resulted in an excellent combination of fine scale filtration efficiency and high permeability [51]. 


\subsection{Membrane characterization}

The morphology of prepared membranes was characterized by the Scanning electron microscopy (SEM) using Hitachi TM-1000 and Hitachi S-5500 instruments (Japan) for preliminary and detailed analysis, respectively. The SEM images in Fig. 3 show that a membrane consist of randomly oriented alumina nanofibers with the length of $0.5-1 \mu \mathrm{m}$. The TEM images obtained by the Hitachi HT-7700 instrument (Japan) confirm the formation of several carbon layers on the nanofibers with the total thickness of around 1-2 nm (insert in Fig. 3b).

$\mathrm{X}$-ray fluorescent microanalysis using combined energy dispersive X-ray spectrophotometer Quantax 70 (Bruker, Germany) with silicon drift detector (SDD) showed that the carbon distribution is uniform along the entire cross-section of $\mathrm{C}-\mathrm{Nafen}$ membrane.

The carbon structure formation on Nafen membranes was also studied by Raman spectroscopy using Horiba Jobin Yvon 64000 triple spectrometer (France). The relative intensity of $\mathrm{D}$ and $\mathrm{G}$ peaks in $\mathrm{C}-\mathrm{Nafen}$ membrane spectrum was approximately 1.035 , which indicated the formation of disordered structures of amorphous carbon during the CVD synthesis.

The Four Probe DC method was employed for measurement of electrical resistance. The Nafen membrane demonstrated high resistance of $2.3 \mathrm{M} \Omega \cdot \mathrm{m}$, while the C-Nafen membrane resistance was very low $(0.002 \Omega \cdot \mathrm{m})$, which is typical for conductive carbon layers.

Thermal analysis of C-Nafen membranes was performed using Jupiter 449 (Netzsch, Germany) with mass-spectrometer detector. It was determined that the membranes contains 68.9 $\%$ of alumina, $19.6 \%$ of carbon structures including $4.5 \%$ of oxygen and nitrogen containing functional groups, and $11.5 \%$ of adsorbed water.

Membrane pore and surface area characterization was done by low temperature nitrogen adsorption experiments using ASAP-2420 (Micromeritics, USA). The Nafen membrane is characterized by the porosity of $75 \%$, specific surface area of $146 \mathrm{~m}^{2} / \mathrm{g}$, and maximum of pore diameter distribution curve at $28 \mathrm{~nm}$. The corresponding parameters of C-Nafen membrane are $62 \%, 107 \mathrm{~m}^{2} / \mathrm{g}$, and $16 \mathrm{~nm}$. Both types of membranes are hydrophilic.

The cyclic voltammetry in $0.1 \mathrm{M} \mathrm{KCl}$ solution confirmed that the carbon coated $\mathrm{C}-\mathrm{Nafen}$ membranes are ideally polarizable in the range of applied voltages from $-0.5 \mathrm{~V}$ to $+0.8 \mathrm{~V}$. The Potentiostat PI-50 Pro (ELINS Ltd., Russia) was used to perform the measurements.

\subsection{Membrane potential measurement}

The membrane potential is measured in a laboratory made electrochemical cell. It consists of two compartments, between which the membrane is clamped with the help of connection rods and nuts. The cell body is made of chemically inert polytetrafluoroethylene (PTFE), which allows working with a wide range of chemicals. In each of the half-cells, reference $4.2 \mathrm{M}$ $\mathrm{Ag} / \mathrm{AgCl}$ electrode is located. Electrodes are connected to the input of a potentsiostat PI-50 Pro (ELINS Ltd., Russia), which measures the electromotive force (EMF) of the cell in 'broken circuit potential measurement' mode. The input impedance of this device is $10^{12} \Omega$. To prevent the electrical noise, the cell and electrodes are shielded in a metallic box.

The measurements were performed in $\mathrm{KCl}$ and $\mathrm{NaCl}$ aqueous solution. At first, the solution with the fixed concentration $C_{R}$ is placed in both half-cells. The system is kept at room temperature of $25^{\circ} \mathrm{C}$ during 12 hours. The measurements are performed by increasing the electrolyte concentration in the left half-cell by consecutive addition of the concentrate $(1 \mathrm{M}$ or $4.2 \mathrm{M})$. For large values of $C_{L}(>1 \mathrm{M})$, the solution is completely replaced with that of higher concentration. After each addition or replacement, the system is allowed to equilibrate during 30 minutes. Then, the cell EMF is measured. After each series of experiments, the membrane is placed in deionized water for 24 hours to remove the rest of electrolyte solution from the pores. 


\section{Results and discussion}

\subsection{Physical parameters}

Let us start with the description of physical parameters used in the theoretical calculations. For aqueous $\mathrm{KCl}$ solution, the diffusion coefficients are $D_{+}=1.957 \cdot 10^{-9} \mathrm{~m}^{2} / \mathrm{s}$ for $\mathrm{K}^{+}$ion and $D_{-}=2.032 \cdot 10^{-9} \mathrm{~m}^{2} / \mathrm{s}$ for $\mathrm{Cl}^{-}$ion. For aqueous $\mathrm{NaCl}$ solution, the diffusion coefficient of $\mathrm{Na}^{+}$ ion is $D_{+}=1.330 \cdot 10^{-9} \mathrm{~m}^{2} / \mathrm{s}$. The density and dynamic viscosity of ionic solutions are taken as those of water: $\rho=997 \mathrm{~kg} / \mathrm{m}^{3}$ and $\mu=0.888 \cdot 10^{-3} \mathrm{~Pa} \cdot \mathrm{s}$. The other parameters used are as follows: $T=298.15 \mathrm{~K}, R=8.314 \mathrm{~J} /(\mathrm{mol} \mathrm{K}), F=96485 \mathrm{C} / \mathrm{mol}, \varepsilon=78.49, \varepsilon_{0}=8.854 \cdot 10^{-12}$ $\mathrm{F} / \mathrm{m}$.

For comparison between experimental data and theoretical calculations based on the Space-Charge model, we take the pore radius as $R_{p}=14 \mathrm{~nm}$ for Nafen membrane and $R_{p}=8$ $\mathrm{nm}$ for $\mathrm{C}-$ Nafen membrane, while the pore length is $L_{p}=400 \mu \mathrm{m}$. When the results of SpaceCharge and Navier-Stokes / Nernst-Planck-Poisson models are compared, the pore radius is $R_{p}=8 \mathrm{~nm}$, while the length is reduced to $L_{p}=4 \mu \mathrm{m}$ since very long pores cannot be easily handled by the latter model. The corresponding dimensions of reservoirs are $R_{r}=100 \mathrm{~nm}$, $L_{r}=400 \mathrm{~nm}$, see Fig. 1. By comparing scalings (12) and (19), one can see that the decrease of pore length by a number of times results in the increase of velocity and ion fluxes by the same number of times.

\subsection{The fields of potential, concentration, and pressure}

The comparison between non-polarizable and polarizable uncharged nanopores is shown in Fig. 4 for $\mathrm{NaCl}$ aqueous solution. In the former case described by Eqs. (5) and (6), the concentrations of cations and anions coincide. Due to the difference between ion diffusion coefficients $\left(D_{+} / D_{-}=0.65\right)$, the electric field $E=-\nabla \Phi$ develops. It speeds up the slower diffusing cation and retards the faster diffusing anion to make the total ion fluxes equal and satisfy the condition of zero current. In a polarizable pore, this electric field induces the surface charge, which changes almost linearly from the pore entrance $\left(Z / L_{p}=0\right)$ to the pore exit $\left(Z / L_{p}=1\right)$, while keeping the total surface charge $\bar{\sigma}$ zero, see Fig. 4 (d). It results in the higher concentration of cations (anions) at negatively (positively) charged part of the pore, Fig. 4 (b). The separation of charge induces the Donnan potentials at the pore entrance and exit, which both contribute to the enhancement of membrane potential in comparison with non-polarizable pore, Fig. 4 (a). The separation of charge also results in osmotic pressure jumps at the pore entrance and exit, see Fig. 4 (c). These jumps balance the electric force, which develops in the interfacial regions, where non-zero net charge is subjected to a large electric field. A good agreement between the NS-NPP model and the extension of SC model to polarizable nanopores is observed, which validates the latter model.

A closer look at the interfacial regions is presented in Fig. 5. The potential and concentration drops in the NS-NPP model, which takes into account the reservoirs, are smooth and lower in comparison with the SC model, which uses jump boundary conditions. It explains a slight decrease of pressure jump magnitude at the pore exit, see Fig. 4 (c). In a polarizable nanopore, the electric field resulting from strong potential gradients at the entrance and exit significantly increases the induced surface charge there (Figs. $5(\mathrm{c}, \mathrm{d})$ ). This effect can be described only by NS-NPP model with continuous change of potential and ion concentrations at the two interfaces.

The two-dimensional fields of potential, ion concentrations, and pressure without interfacial jumps are presented in Fig. 6. They are calculated by the SC model. It can be seen that the surface potential is constant $(-2.7 \mathrm{mV})$, while the potential increases (decreases) in those regions of pore, where the concentration of cations (anions) is higher. The pressure rise near 

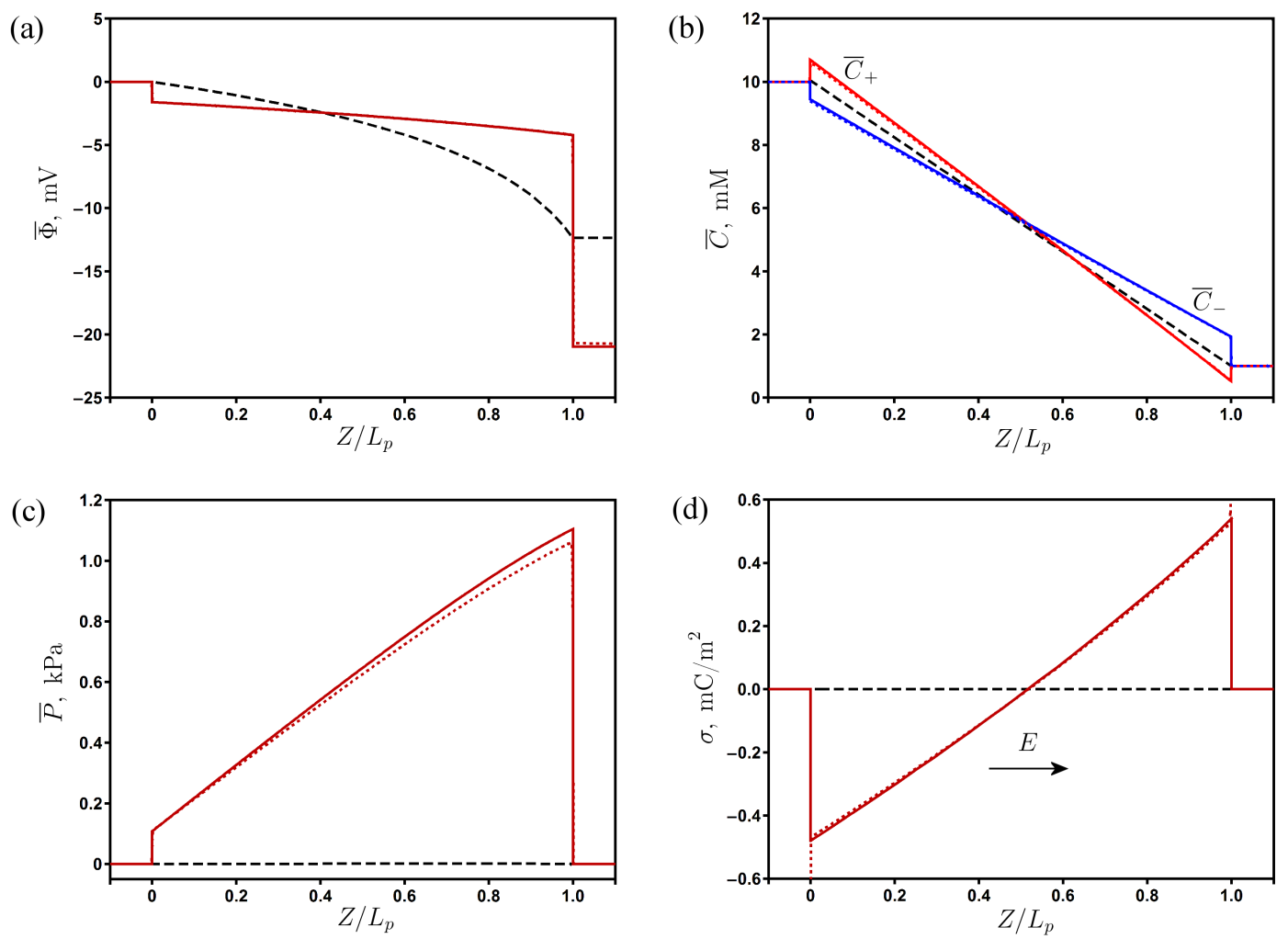

Figure 4: The average potential (a), ion concentrations (b), pressure (c), and surface charge density (d) for nonpolarizable $(\sigma=0$, dashed curves) and polarizable $(\bar{\sigma}=0$, solid curves) nanopores in NaCl solution calculated by the SC model and NS-NPP model (dotted curves). $R_{p}=8 \mathrm{~nm}, L_{p}=4 \mu \mathrm{m}, C_{L}=10 \mathrm{mM}, C_{R}=1 \mathrm{mM}$.


Figure 5: The average potential (a), ion concentrations (b), and surface charge density at the pore interfaces $(\mathrm{c}, \mathrm{d})$ for non-polarizable $(\sigma=0$, dashed curves) and polarizable $(\bar{\sigma}=0$, solid curves) nanopores calculated by the SC model and NS-NPP model (dotted curves). For parameters, see Fig. 4. 

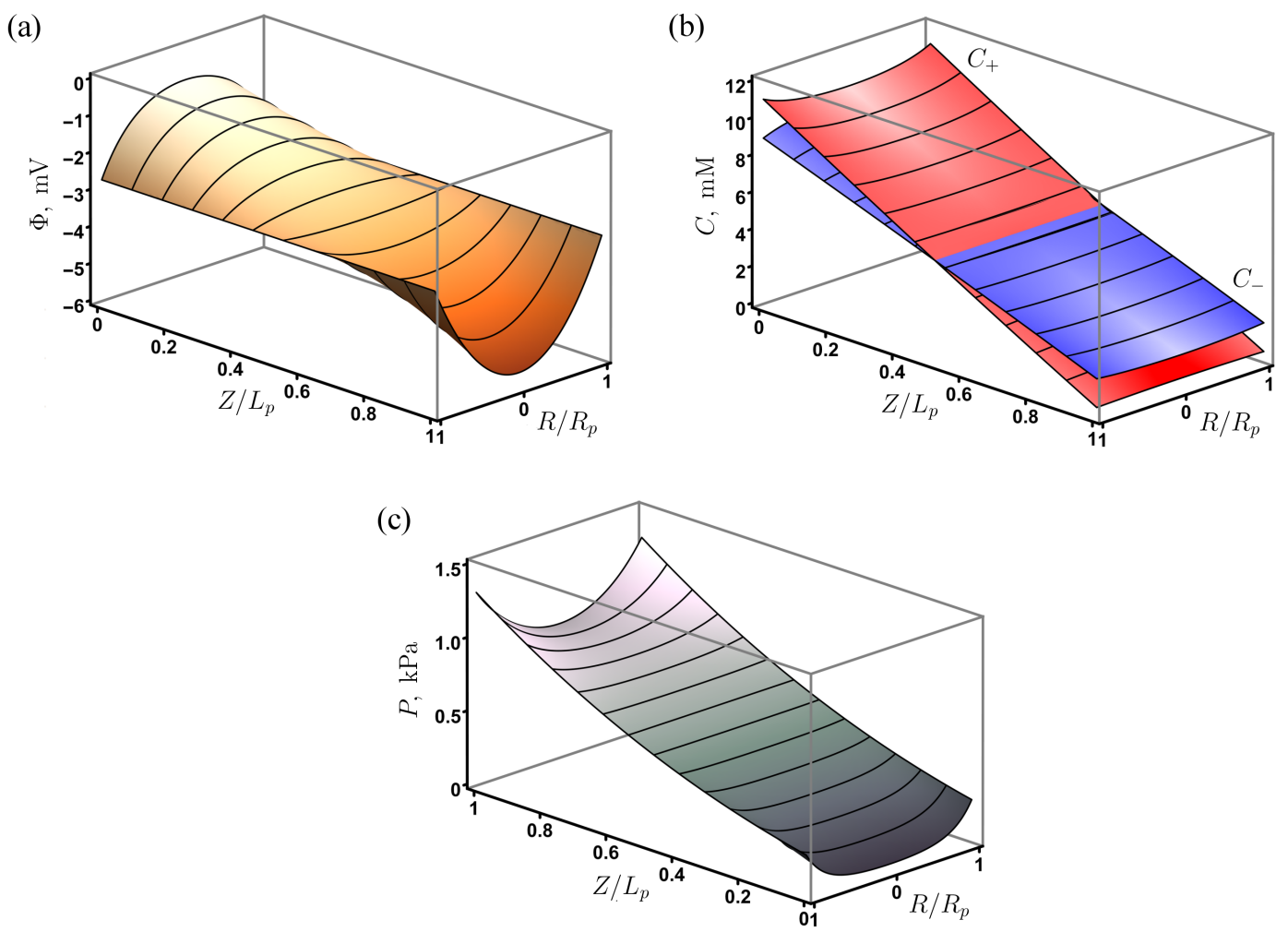

Figure 6: The fields of potential (a), ion concentrations (b), and pressure (c) in a polarizable nanopore with $\bar{\sigma}=0$ in aqueous $\mathrm{NaCl}$ solution calculated by the $\mathrm{SC}$ model. For parameters, see Fig. 4. The direction of axial coordinate is reversed in (c).

(a)

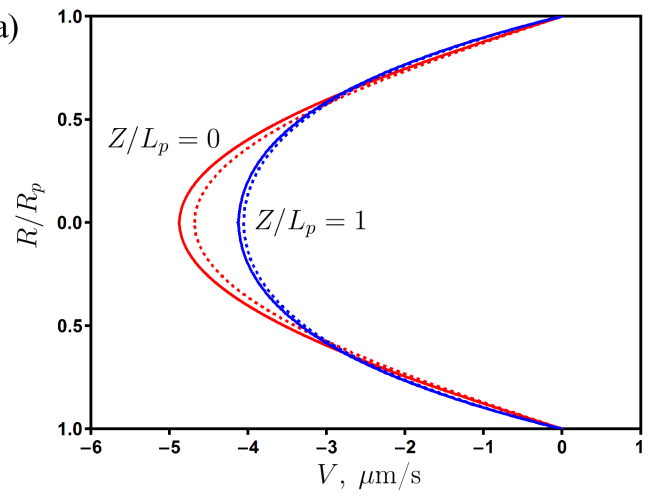

(c)

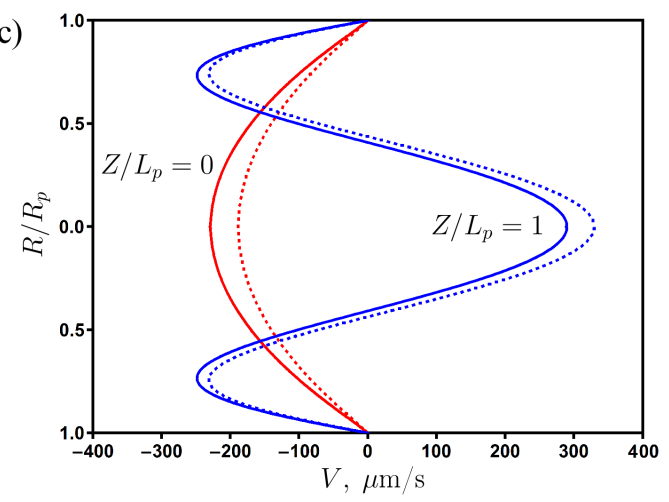

(b)

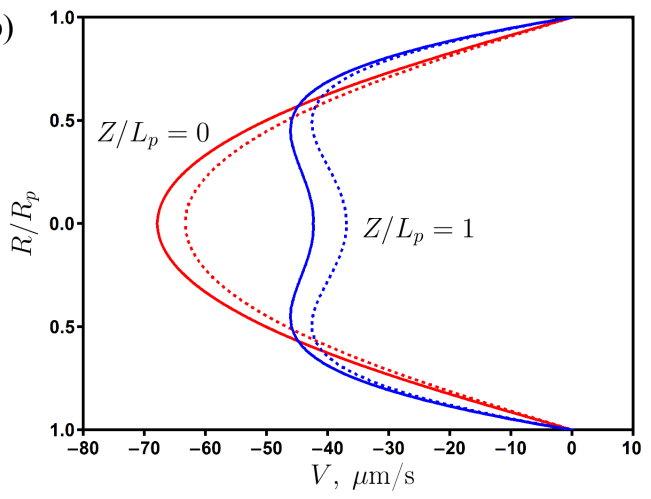

(d)

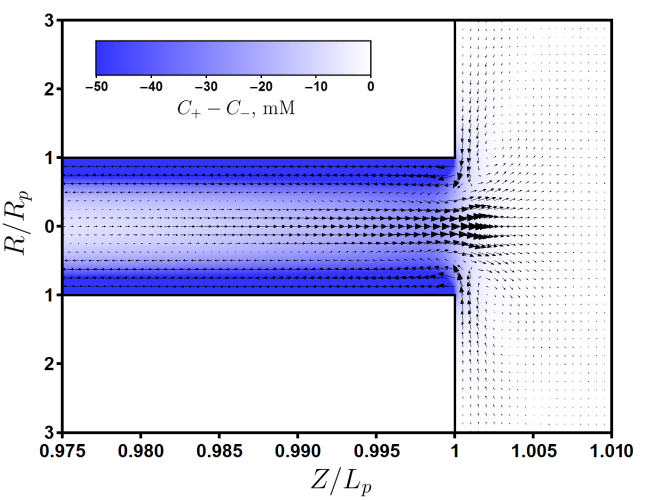

Figure 7: The axial velocity profiles at the pore entrance and exit for $C_{L}=10 \mathrm{mM}$ (a), $C_{L}=100 \mathrm{mM}$ (b), $C_{L}=1000 \mathrm{mM}(\mathrm{c})$, and $C_{R}=1 \mathrm{mM}$. SC model (solid curves) and NS-NPP model (dotted curves). Velocity and volume charge fields at the pore exit for $C_{L}=1000 \mathrm{mM}, C_{R}=1 \mathrm{mM}$ (d). $R_{p}=8 \mathrm{~nm}, L_{p}=4 \mu \mathrm{m}$. 
the wall compensates the electric force (the last term in Eq. (13)), which is directed towards the wall.

\subsection{The induced-charge electroosmosis}

A higher osmotic pressure jump at the pore exit in comparison with that at the pore entrance induces the osmotic flow in the direction from lower to higher salt concentration, Fig. 7 (a). The higher concentration of cations (anions) near the pore entrance (exit) results in the decrease (increase) of velocity in the near-wall region due to the presence of electric field. The opposite situation is observed at the pore center since the total volume flow rate (average velocity) is constant along the pore. The described effect becomes stronger with increasing the concentration contrast, Fig. 7 (b,c). In this case, the induced charge at the pore wall increases resulting in a higher charge separation inside the pore, and, consequently, higher electroosmotic flow in the near-wall region. For high concentration contrast $\left(C_{L} / C_{R}=1000\right)$, a region of large negative charge develops at the pore exit. The flow near the pore walls enhances, while its reversal is observed at the pore center to keep the average axial velocity constant, see Figs. 7 $(\mathrm{c}, \mathrm{d})$. Two counter-rotating vortices develop occupying around $2.5 \%$ of the total channel length and extending to the right reservoir. Note that a similar effect was found to be responsible for overlimiting current when applying a potential difference across a microchannel [52]. In this work, we show that electroosmotic flow can be generated by the interaction between electric field resulting from diffusion of ions with different mobilities and charge separation caused by the induced surface charge.

The comparison between the SC and NS-NPP models in Fig. 7 reveals that the latter model provides slightly reduced velocity magnitudes. This effect becomes more noticeable with increasing the concentration contrast due to stronger reduction of osmotic pressure drop at the pore exit in the NS-NPP model, see Fig. 4 (c). The calculations show that the differences between models become smaller with increasing the pore length. Note that the velocity profiles in the NS-NPP model were taken at $Z / L_{p}=0.005$ and $Z / L_{p}=0.995$ to avoid the influence of entrance/exit effects.

\subsection{Comparison between theory and experiment}

The membrane potentials of uncharged non-polarizable $(\sigma=0, R=14 \mathrm{~nm})$ and polarizable $(\bar{\sigma}=0, R=8 \mathrm{~nm})$ membranes in $\mathrm{KCl}$ and $\mathrm{NaCl}$ solutions have been calculated with the help of SC model. The results are shown in Fig. 8 by dashed curves. For non-polarizable pores, the membrane potential linearly depends on the logarithm of concentration ratio according to (7). For polarizable pores, it significantly deviates from linear dependence since the magnitude of induced charge increases with increasing the concentration contrast. In particular, for $\mathrm{NaCl}$ solution with $\log \left(C_{L} / C_{R}\right)=1$, the induced charge varies from -0.48 to $0.54 \mathrm{mC} / \mathrm{m}^{2}$ (Fig. 4 (d)), while for $\log \left(C_{L} / C_{R}\right)=3$ its variation is from -8.58 to $8.06 \mathrm{mC} / \mathrm{m}^{2}$. The induced-charge enhancement of diffusion potential for $\mathrm{KCl}$ solution with almost equal ion diffusion coefficients $\left(D_{+} / D_{-}=0.96\right)$ is quite significant (more than 16 times at $\log \left(C_{L} / C_{R}\right)=3$ ), see Fig. 8 (a). For $\mathrm{NaCl}$ solution with $D_{+} / D_{-}=0.65$, the enhancement is around 2.6 times at the same concentration contrast, see Fig. 8 (b).

The theoretical results are well supported by the experimental data. The measurements for Nafen membrane were performed at $\mathrm{pH}=9.1$ in $\mathrm{KCl}$ solution and $\mathrm{pH}=8$ in $\mathrm{NaCl}$ solution, which correspond to the point of zero charge for alumina surface $[53,54]$. The values of surface charge density $\sigma$ obtained by fitting of experimental data to the theoretical model of nonpolarizable nanopore with $R=14 \mathrm{~nm}$ are presented in Table 2. They are rather low, so the fitted curves only slightly deviate from those corresponding to $\sigma=0$.

For C-Nafen membrane with conductive carbon surface, the adsorption of alkali metal 
(a)



(b)

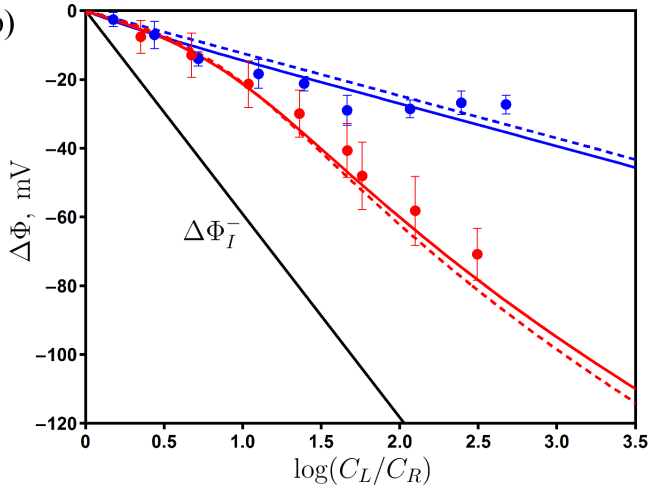

Figure 8: Membrane potential of Nafen (blue) and C-Nafen (red) membranes in $\mathrm{KCl}$ (a) and $\mathrm{NaCl}$ (b) aqueous solutions. Experimental data (points), calculations for uncharged non-polarizable (blue) and polarizable (red) pores (dashed curves), fitting of experimental data (solid curves), ideal anion selectivity (solid black line). Error bars correspond to 1 standard deviation. The parameters are given in Table $2, L_{p}=400 \mu \mathrm{m}$.

Table 2: Experimental cases with fitted values of $\sigma$ (Nafen membranes) or $R$ and $\bar{\sigma}$ (C-Nafen membranes).

\begin{tabular}{lcccc}
\hline \hline Electrolyte & \multicolumn{2}{c}{ Aqueous $\mathrm{KCl}$} & \multicolumn{2}{c}{ Aqueous $\mathrm{NaCl}$} \\
Membrane & Nafen & C-Nafen & Nafen & C-Nafen \\
\hline$C_{R}, \mathrm{mM}$ & 1 & 0.1 & 1 & 1 \\
$R, \mathrm{~nm}$ & 14 & 8.8 & 14 & 9.4 \\
$\sigma$ or $\bar{\sigma}, \mathrm{mC} / \mathrm{m}^{2}$ & 0.329 & 0.039 & 0.128 & 0.045 \\
\hline \hline
\end{tabular}

cations on the defects of carbon structure can occur and modify the surface charge, see [48] and [55-57]. To minimize this effect, low electrolyte concentrations were used: $C_{R}=0.1 \mathrm{mM}$ for $\mathrm{KCl}$ and $C_{R}=1 \mathrm{mM}$ for $\mathrm{NaCl}$. In this case, the experimental data were fitted to theoretical model of polarizable nanopore to obtain the total surface charge density $\bar{\sigma}$ and pore radius $R$, see Table 2. The obtained values of $\bar{\sigma}$ are positive but rather small, while the $R$ values are in good agreement with low temperature nitrogen adsorption data $(8 \mathrm{~nm})$ [48]. So, the fitted curves are close to those of fully uncharged $(\bar{\sigma})$ polarizable membranes in Fig. 8.

\subsection{The influence of fixed concentration $C_{R}$ and pore radius}

The diffusion potential $\Delta \Phi_{D}$ of an uncharged non-polarizable membrane at constant temperature depends only on the ratio of concentrations and ion diffusion coefficients, see Eq. (7) and the corresponding dashed curve in Fig. 9 (a). In this case, there are no interfacial pressure jumps, so the average axial velocity $\bar{V}=0$ (Fig. $9(\mathrm{~b})$ ). The average ion flux $\bar{J}=J$, where $J$ is given by (6). Figure 9 (c) shows that it increases with increasing the concentration contrast. The magnitude of pore-averaged potential increases as well according to (9) since stronger electric field is required to make the ion fluxes equal at higher concentration contrast (Fig. 9 (d)).

In the case of membrane with polarizable pores, the situation is strikingly different. The membrane potential now essentially depends on the concentration $C_{R}$. At lower concentrations, the Debye length is larger, which means a stronger overlap of electric double layers created by the induced surface charge. The calculations reveal that the magnitude of Donnan potentials at the pore entrance and exit (see Fig. 4 (a)) becomes larger when $C_{R}$ decreases at fixed ratio $C_{L} / C_{R}$. It explains the stronger enhancement of membrane potential at lower $C_{R}$ shown in Fig. 9 (a). The magnitudes of average axial velocity and average ion flux increase with increasing 

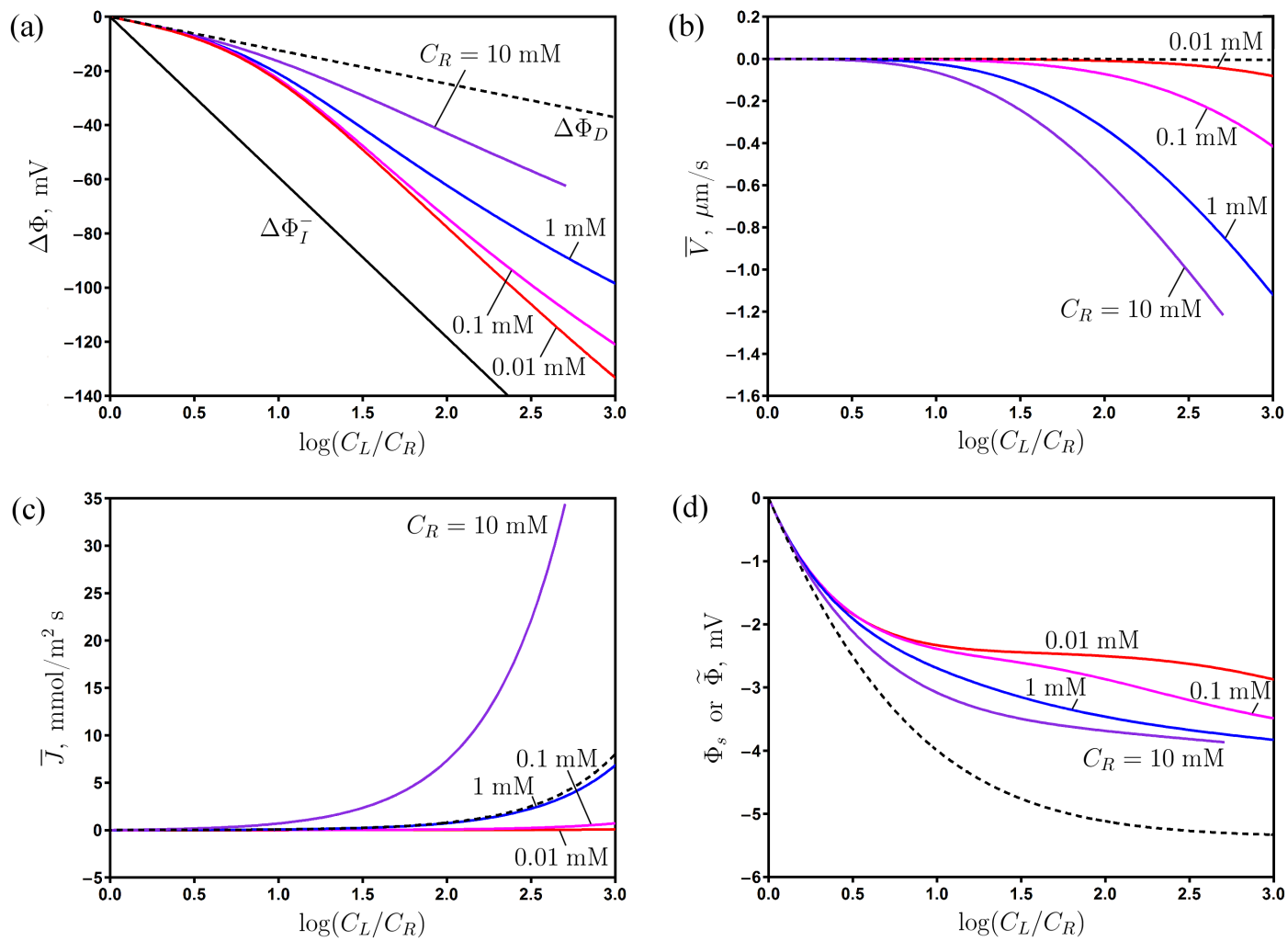

Figure 9: The membrane potential (a), average axial velocity (b), average ion flux (c), and surface or poreaveraged potential (d) for different values of concentration $C_{R}$ in $\mathrm{NaCl}$ aqueous solution. Uncharged nonpolarizable (dashed curve, $C_{R}=1 \mathrm{mM}$ ) and polarizable (solid curves) nanopores. $R_{p}=8 \mathrm{~nm}, L_{p}=400$ $\mu \mathrm{m}$.


Figure 10: The membrane potential (a), average axial velocity (b), average ion flux (c), and surface or poreaveraged potential (d) for different values of pore radius $R_{p}$ in $\mathrm{NaCl}$ aqueous solution. Uncharged nonpolarizable (dashed curve) and polarizable (solid curves) nanopores. $C_{R}=1 \mathrm{mM}, L_{p}=400 \mu \mathrm{m}$. 

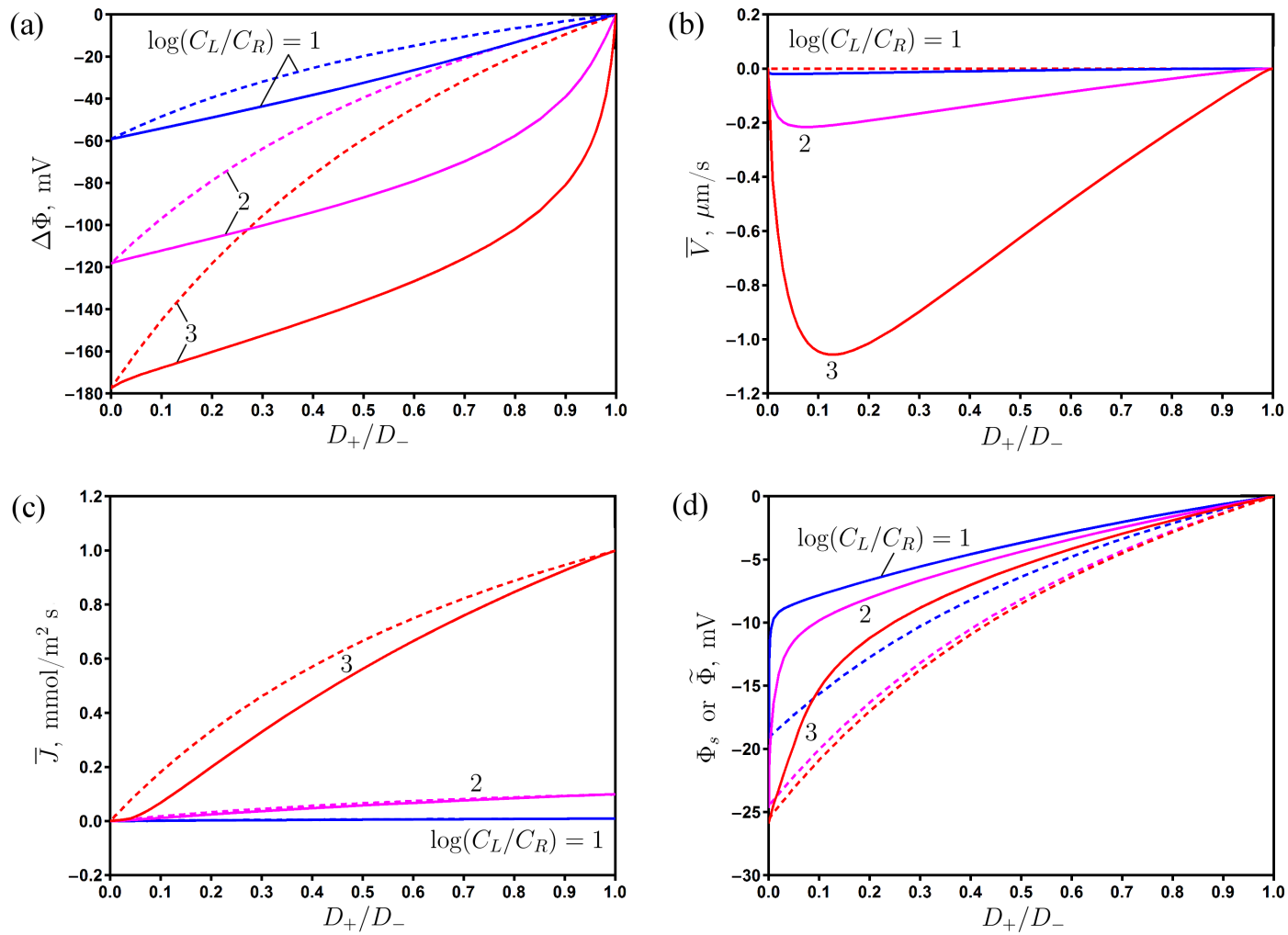

Figure 11: The dependence of membrane potential (a), average axial velocity (b), average ion flux (c), and surface or pore-averaged potential (d) on ion diffusion coefficients ratio in $\mathrm{NaCl}$ aqueous solution. Uncharged non-polarizable (dashed curves) and polarizable (solid curves) nanopores. $C_{R}=0.1 \mathrm{mM}, R_{p}=8 \mathrm{~nm}, L_{p}=400$ $\mu \mathrm{m}, D_{-}=2 \cdot 10^{-9} \mathrm{~m}^{2} / \mathrm{s}$.

$C_{R}$, see Fig. 9 (b,c). The redistribution of surface charge by electric field causes its suppression near the pore surface (Fig. 6 (a)). Thus, the magnitude of surface potential $\Phi_{s}$ in a polarizable nanopore becomes smaller than that in a non-polarizable nanopore, see Fig. 9 (d). Note that in the latter case, the surface potential is not constant, so the value $\Phi_{s}$ is compared with the pore-averaged potential $\widetilde{\Phi}$ given by (9). It is clear that the suppression of electric field becomes stronger for lower concentrations $C_{R}$.

The effect of decreasing the pore radius is similar to that of decreasing concentration. It is demonstrated in Fig. 10. In both cases, the Debye length increases leading to a stronger overlap of electric double layers. The membrane potential shows a significant increase when $R_{p}$ decreases, but even at large $R_{p}$ its enhancement is quite noticeable in comparison with the non-polarizable case. The variation of pore radius, however, does not influence the average ion flux, see Fig. 10 (c).

\subsection{The influence of ion diffusion coefficients ratio}

The effect of ion diffusion coefficients ratio on the membrane potential is shown in Fig. 11 (a). For both non-polarizable and polarizable pores, $\Delta \Phi=0$ when $D=D_{+} / D_{-}=1$, while it approaches the Nernst potential when $D \rightarrow 0$, see Eqs. (7) and (8). In the range $0<D<1$, a dramatic enhancement of diffusion potential in a polarizable pore is observed. It becomes larger with increasing the concentration contrast. Especially unusual is the strong rise of membrane potential magnitude near $D=1$. It means that a very small difference between diffusion coefficients can result in a large change of membrane potential. This conclusion is confirmed by the experimental data in $\mathrm{KCl}$ solution, see Fig. 8 (a).

For a polarizable nanopore, the average axial velocity is zero when $D=1$ since the 
interfacial pressure jumps are not formed in the absence of induced charge (Fig. 11 (b)). It also tends to zero when $D \rightarrow 0$. The calculations show that in this case the magnitude of induced charge near the pore entrance becomes much larger than near the pore exit, which results in almost equal magnitudes of interfacial pressure jumps. An interesting conclusion is that the magnitude of average axial velocity reaches maximum at some value of diffusion coefficients ratio.

The average ion flux decreases with decreasing the ratio of diffusion coefficients $D=$ $D_{+} / D_{-}$at fixed $D_{-}$, see Fig. 11 (c). According to (6), the flux is dominated by the smaller diffusivity. At the same time, the magnitude of pore-averaged potential described by Eq. (9) increases when the ratio $D_{+} / D_{-}$goes to zero (Fig. $11(\mathrm{~d})$ ). The decrease of surface potential magnitude in a polarizable nanopore in comparison with the pore-averaged potential of a nonpolarizable nanopore is clearly seen. Note that the average axial velocity, ion flux, and surface potential become larger in magnitude when the concentration contrast becomes larger.

The calculations in this work have been performed assuming that $D_{+}<D_{-}$, so $0<D<1$. The case $D_{+}>D_{-}$can be reduced to the previously considered one by applying the following change of dimensionless variables

$$
\begin{gathered}
\boldsymbol{u} \rightarrow \text { Du }, \quad c_{+} \rightarrow c_{-}, \quad c_{-} \rightarrow c_{+}, \quad \varphi \rightarrow-\varphi, \\
\sigma_{s} \rightarrow-\sigma_{s}, \quad \varphi_{s} \rightarrow-\varphi_{s}, \quad q_{s} \rightarrow-q_{s} .
\end{gathered}
$$

In (36), one should choose either the first, second, or third formula when boundary conditions C1, C2, or C3 are imposed on the nanopore wall, respectively, see Table 1. After application of (35), (36), the governing equations (13)-(17) and boundary conditions in Table 1 remain the same, but in formulas for dimensional variables (12) and dimensionless parameters (18) $D_{-}$is replaced by $D_{+}$and vice versa. So, one finds $0<D<1$ as in the previously considered case. It follows that at the same ratio of smaller to larger diffusivity, the magnitude of membrane potential is the same, but its sign is negative when $D_{+}<D_{-}$and positive when $D_{+}>D_{-}$.

\section{Conclusion}

In this paper, we have studied theoretically and experimentally the generation of membrane potential at zero current in nanoporous membranes separating two reservoirs with different electrolyte concentrations. The TMS model was used to describe the transport of ions in uncharged non-polarizable nanopores. The simulation of polarizable nanopores was performed by the Navier-Stokes / Nernst-Planck / Poisson (NS-NPP) model with floating boundary condition at the conductive pore wall. The extension of the Space-Charge (SC) model to polarizable nanopores was proposed for the first time and validated against the full NS-NPP model.

A new mechanism for the generation of membrane potential in polarizable nanoporous membranes has been discovered. The electric field generated by diffusion of ions with different mobilities induces a non-uniform surface charge, which results in charge separation inside the nanopore. A higher concentration of cations (anions) is observed at negatively (positively) charged part of the pore. The corresponding Donnan potentials appear at the pore entrance and exit leading to a dramatic enhancement of membrane potential in comparison with uncharged dielectric membrane. The theoretical predictions have been confirmed experimentally by measuring the membrane potential of dielectric and conductive membranes in $\mathrm{KCl}$ and $\mathrm{NaCl}$ aqueous solutions. The membranes were prepared from Nafen alumina nanofibers with the diameter of around $10 \mathrm{~nm}$. Chemical vapor deposition (CVD) was used to form conductive carbon layers on the nanofibrous membrane structure.

The calculations reveal that charge separation induces unequal pressure jumps at the pore entrance and exit leading to osmotic flow in the direction from lower to higher concentration. At 
high concentration contrast, the interaction between electric field and uncompensated charge results in the appearance of electrokinetic flow near the pore exit in the form of counter-rotating vortices. A detailed simulation by NS-NPP model reveals that the magnitude of induced charge increases greatly in the interfacial regions due to strong electric fields there.

It is found that the enhancement of membrane potential in initially uncharged polarizable nanopores becomes greater with decreasing the electrolyte concentration and pore radius. The membrane potential is zero at equal ion diffusion coefficients and coincides with the Nernst potential when their ratio tends to zero (or to infinity) for both non-polarizable and polarizable nanopores. In the intermediate region, the latter exhibit much large membrane potential in comparison with the former ones. A strong sensitivity of membrane potential to small differences in ion diffusion coefficients is demonstrated. The described phenomenon may find applications in precise determination of ion mobilities, electrochemical and bio-sensing, as well as design of nanofluidic and bioelectronic devices.

\section{Acknowledgement}

This work is supported the Russian Science Foundation, Project 15-19-10017. The physicochemical analysis of materials was carried out on equipment of Krasnoyarsk Scientific Center of Shared Facilities SB RAS.

\section{Appendix}

The formulas for coefficients of matrix $\mathcal{L}$ were derived in [15] for the case of equal ion diffusion coefficients $\left(D=D_{+} / D_{-}=1\right)$. We have generalized these formulas to the case when $D_{+}$and $D_{-}$have different values:

$$
\begin{gathered}
\mathcal{L}_{11}=\frac{1}{8 \alpha} \\
\mathcal{L}_{22}=2 c_{v} \int_{0}^{1} r(D \exp (\psi(r))+\exp (-\psi(r))) d r- \\
-\frac{16 c_{v}^{2}}{\alpha} \int_{0}^{1}\left[r \cosh (\psi(r)) \ln r\left(\frac{r^{2}}{2} \cosh (\psi(r))-\frac{\lambda^{2}}{4 c_{v}}\left(r \frac{\partial \psi(r)}{\partial r}\right)^{2}\right)\right] d r \\
\mathcal{L}_{33}=-\frac{8 c_{v}}{\alpha} \int_{0}^{1} r\left[\sinh (\psi(r)) \lambda^{2}\left(\psi(r)-\psi_{s}\right)-\frac{\alpha}{4}(D \exp (\psi(r))+\exp (-\psi(r)))\right] d r \\
\mathcal{L}_{12}=\mathcal{L}_{21}=\frac{c_{v}}{\alpha} \int_{0}^{1}\left(r-r^{3}\right) \cosh (\psi(r)) d r \\
\mathcal{L}_{13}=\mathcal{L}_{31}=\frac{4}{\alpha} \int_{0}^{1} r \lambda^{2}(\psi(r)-\psi s) d r \\
\mathcal{L}_{23}=\mathcal{L}_{32}=\frac{8 c_{v}}{\alpha} \int_{0}^{1} r\left[\cosh (\psi(r)) \lambda^{2}\left(\psi(r)-\psi_{s}\right)-\frac{\alpha}{4}(D \exp (\psi(r))-\exp (-\psi(r)))\right] d r
\end{gathered}
$$

where $\psi_{s}=\psi(1, z)$. Note that the dependence of $\psi$ and $c_{v}$ on $z$ is not explicitly stated here.

\section{References}

[1] H. Strathmann. Introduction to membrane science. Wiley-VCH, 2011. 
[2] Y. Tanaka, Ion Exchange Membranes: Fundamentals and Applications, Elsevier, Amsterdam, 2015.

[3] A.B. Yaroslavtsev, V.V. Nikonenko, V.I. Zabolotsky, Ion transfer in ion-exchange and membrane materials, Russ. Chem. Rev. 72 (5) 393-421 (2003).

[4] F.G. Bănică, Chemical Sensors and Biosensors: Fundamentals and Applications, John Wiley \& Sons, Chichester, UK, 2012.

[5] H. Strathmann, Electrodialysis, a mature technology with a multitude of new applications, Desalination 264 (2010) 268-288.

[6] A. Cipollina, G. Micale, Sustainable Energy from Salinity Gradients, Elsevier/Woodhead Publishing, 2016.

[7] J. Malmivuo, R. Plonsey, Bioelectromagnetism - Principles and Applications of Bioelectric and Biomagnetic Fields, Oxford University Press, New York, 1995.

[8] M. Yangand, W.J. Brackenbury, Membrane potential and cancer progression, Front. in Physiology, 4 (2013) 185.

[9] T. Teorell, An attempt to formulate a quantitative theory of membrane permeability, Proc. Soc. Exp. Biol. Med. 33 (1935) 282-285.

[10] K.H. Meyer, J.F. Sievers. La perméabilité des membranes I. Théorie de la perméabilité ionique. Helv. Chim. Acta 19 (1936) 649-664.

[11] A.H. Galama, J.W. Post, H.V.M. Hamelers, V.V. Nikonenko, P.M. Biesheuvel, J. Membr. Sci. Res. 2 (2016) 128-140.

[12] Y. Lanteri, A. Szymczyk, P. Fievet, Influence of steric, electric, and dielectric effects on membrane potential, Langmuir 24 (2008) 7955-7962.

[13] I. Makra, G. Jágerszki, I. Bitter, R.E. Gyurcsányia, Nernst-Planck/Poisson model for the potential response of permselective gold nanopores, Electrochim. Acta, 73 (2012) 70-77.

[14] R.J. Gross, J.F. Osterle, Membrane transport characteristics of ultrafine capillaries. J. Chem. Phys. 49 (1968) 228-234.

[15] P.B. Peters, R. van Roij, M.Z. Bazant, P.M. Biesheuvel, Analysis of electrolyte transport through charged nanopores. Phys. Rev. E 93 (2016) 053108.

[16] G.B. Westermann-Clark, J.L. Anderson, Experimental verification of the space-charge model for electrokinetics in charged microporous membranes. J. Electrochem. Soc. 130 (1983) 839-847.

[17] G.B. Westermann-Clark, C.C. Christoforou, The exclusion-diffusion potential in charged porous membranes, J. Electroanal. Chem. 198 (1986) 213-231.

[18] P. Fievet, B. Aoubiza, A. Szymczyk, J. Pagetti, Membrane potential in charged porous membranes, J. Membr. Sci. 160 (1999) 267-275.

[19] W.J. Shang, X.L. Wang, Y.X. Yu, Theoretical calculation on the membrane potential of charged porous membranes in 1-1, 1-2, 2-1 and 2-2 electrolyte solutions, J. Membr. Sci. 285 (2006) 362-375.

[20] M.B. Kristensen, A. Bentien, M. Tedesco, J. Catalano, Counter-ion transport number and membrane potential in working membrane systems, J. Coll. Int. Sci. 504 (2017) 800-813. 
[21] H.J.M. Hijnen, J.A.M. Smit, The effect of the pH on electrolyte transport through microporous membranes bearing either weakly or strongly dissociating acid groups. A theoretical analysis using the space-charge model for a cylindrical capillary, J. Membr. Sci. 99 (1995) $285-302$.

[22] J. Schaep, C. Vandecasteele, Evaluating the charge of nanofiltration membranes, J. Membr. Sci. 188 (2001) 129-136.

[23] M.N. Beg, M.A. Matin, Studies with nickel phosphate membranes: evaluation of charge density and test of recently developed theory of membrane potential, J. Membr. Sci. 196 (2002) 95-102.

[24] G.M. Geise, H.J. Cassady, D.R. Paul, B.E. Logan, M.A. Hickner, Specific ion effects on membrane potential and the permselectivity of ion exchange membranes, PCCP 16 (2014) 21673-21681.

[25] E. Kumamoto, Effect of unstirred layers on the membrane potential in a concentration cell, J. Membr. Sci. 9 (1981) 43-51.

[26] M. Tagliazucchi, I. Szleifer, Transport mechanisms in nanopores and nanochannels: can we mimic nature? Mater. Today 18 (2015) 131-142.

[27] Z.S. Siwy, S. Howorka, Engineered voltage-responsive nanopores, Chem. Soc. Rev. 39 (2010) 1115-1132.

[28] X. Hou, W. Guo, L. Jiang, Biomimetic smart nanopores and nanochannels, Chem. Soc. Rev., 40 (2011) 2385-2401.

[29] M. Nishizawa, V.P. Menon, C.R. Martin, Metal nanotubule membranes with electrochemically switchable ion-transport selectivity, Science, 268 (1995) 700-702.

[30] C.R. Martin, M. Nishizawa, K. Jirage, M. Kang, S.B. Lee, Controlling ion-transport selectivity in gold nanotubule membranes, Adv. Mater. 13 (2001), 1351-1362.

[31] M.S. Kang, C.R. Martin. Investigations of potential-dependent fluxes of ionic permeates in gold nanotubule membranes prepared via the template method, Langmuir, 17 (2001) $2753-2759$.

[32] W. Guan, M.A. Reed, Electric field modulation of the membrane potential in solid-state ion channels, Nano Lett. 12 (2012) 6441-6447.

[33] C. Amatore, A.I. Oleinick, I. Svir, Theory of ion transport in electrochemically switchable nanoporous metallized membranes, ChemPhysChem, 10 (2009) 211-221.

[34] P. Ramírez, S. Mafé, A. Alcaraz, J. Cervera, Modeling of pH-switchable ion transport and selectivity in nanopore membranes with fixed charges, J. Phys. Chem. B 107 (2003) 13178-13187.

[35] M.Z. Bazant, M.S. Kilic, B.D. Storey, A. Ajdari. Towards an understanding of induced-charge electrokinetics at large applied voltages in concentrated solutions, Adv. Colloid Interface Sci. 152 (2009) 48-88.

[36] M.Z. Bazant, T.M. Squires, Induced-charge electrokinetic phenomena, Curr. Op. Coll. Inter. Sci. 15 (2010) 203-213.

[37] M.Z. Bazant, T.M. Squires, Induced-charge electrokinetic phenomena: theory and microfluidic application, Phys. Rev. Lett. 92 (2004) 066101. 
[38] S. Gangwal, O.J. Cayre, M.Z. Bazant, O.D. Velev, Induced-charge electrophoresis of metallodielectric particles, Phys. Rev. Lett. 100 (2008) 058302.

[39] S. Rubin, M.E. Suss, P.M. Biesheuvel, M. Bercovici, Induced-charge capacitive deionization: the electrokinetic response of a porous particle to an external electric field, Phys. Rev. Lett. 117 (2016) 234502.

[40] C. Yang, P. Hinkle, J. Menestrina, I.V. Vlassiouk, Z.S. Siwy, Polarization of gold in nanopores leads to ion current rectification, J. Phys. Chem. Lett. 7 (2016) 4152-4158.

[41] C. Zhao, Y. Song, C. Yang. Induced-charge electrokinetics in a conducting nanochannel with broken geometric symmetry: towards a flexible control of ionic transport, Phys. Fluids 27 (2015) 012003.

[42] J.L. Jackson, Charge neutrality in electrolytic solutions and the liquid junction potential, J. Phys. Chem. 78 (1974) 2060-2064.

[43] R. Chein, H. Chen, C. Liao, Investigation of ion concentration and electric potential distributions in charged membrane/electrolyte systems. J. Membr. Sci. 342 (2009) 121-130.

[44] B. Lu, M.J. Holst, J.A. McCammon, Y.C. Zhou. Poisson-Nernst-Planck equations for simulating biomolecular diffusion-reaction processes I: Finite element solutions. J. Comp. Phys. 229 (2010) 6979-6994.

[45] J.F. Pietschmann, M.T. Wolfram, M. Burger, C. Trautmann, G. Nguyen, M. Pevarnik, V. Bayere, Z. Siwy. Rectification properties of conically shaped nanopores: consequences of miniaturization. PCCP 15 (2013) 16917.

[46] I.I. Ryzhkov, A.V. Minakov. Theoretical study of electrolyte transport in nanofiltration membranes with constant surface potential / charge density, J. Membr. Sci. 520 (2016) $515-528$

[47] S. Levine, J.R. Marriott, G. Neale, N. Epstein, Theory of electrokinetic flow in fine cylindrical capillaries at high zeta-potentials, J. Coll. Inter. Sci. 52, 136 (1975).

[48] V.S. Solodovnichenko, D.V. Lebedev, V.V. Bykanova, A.V. Shiverskiy, M.M. Simunin, V.A. Parfenov, I.I. Ryzhkov, Carbon coated alumina nanofiber membrane for selective ion transport, Adv. Engineer. Mater. 20 (2017) 1700244.

[49] Features of Nafen alumina nanofibers, http://www.anftechnology.com/nafen/

[50] V. Su, M. Terehov, B. Clyne, Filtration performance of membranes produced using nanoscale alumina fibers (NAF), Adv. Engineer. Mater. 14 (2012) 1088-1096.

[51] V.M.T. Su, T.W. Clyne, Hybrid filtration membranes incorporating nanoporous silica within a nanoscale alumina fiber scaffold, Adv. Engineer. Mater. 18 (2016) 96-104.

[52] E.V. Dydek, B. Zaltsman, I. Rubinstein, D.S. Deng, A. Mani, M.Z. Bazant, Overlimiting current in a microchannel, Phys. Rev. Lett. 107 (2011) 118301.

[53] M. Tschapek, C. Wasowski, R.M. Torres Sanchez, The p.z.c. and i.e.p. of $\gamma-\mathrm{Al}_{2} \mathrm{O}_{3}$ and $\mathrm{TiO}_{2}$, J. Electroanal. Chem. 74 (1976) 167-176.

[54] R. Sprycha, Electrical double layer at alumina/electrolyte interface, J. Coll. Int. Sci. 127 (1989) 1-11.

[55] G. Mpourmpakis, G. Froudakis, Why alkali metals preferably bind on structural defects of carbon nanotubes: A theoretical study by first principles, J. Chem. Phys. 125 (2006) 204707. 
[56] D.J. Cole, P.K. Ang, K.P. Loh, Ion adsorption at the graphene/electrolyte interface, J. Phys. Chem. Lett. 2 (2011) 1799-1803.

[57] D. Umadevi, G.N. Sastry, Metal ion binding with carbon nanotubes and graphene: effect of chirality and curvature, Chem. Phys. Lett. 549 (2012) 39-43. 